\title{
Study of a finite volume scheme for the regularised mean curvature flow level set equation
}

\author{
Robert Eymard, Angela Handlovičová ${ }^{*}$ and Karol Mikula
}

\begin{abstract}
We propose a new finite volume numerical scheme for the approximation of regularised mean curvature flow level set equations, which ensures the maximum principle, and which is shown to converge to the solution of the problem. The convergence proof uses the monotonicity of the operator, in order to get the strong convergence of the approximation of the gradient. The regularisation of the original level set problem is practically meaningful and not restrictive, especially when dealing with image processing applications. Numerical examples provide indications about the accuracy of the method.
\end{abstract}

Keywords: Regularised mean curvature motion equation, convergence of finite volume method, LerayLions operators.

\section{Introduction}

We consider the following problem: find an approximate solution to the equation

$$
u_{t}-g(|\nabla u|) \operatorname{div}\left(\frac{\nabla u}{f(|\nabla u|)}\right)=r, \text { a.e. }(x, t) \in \Omega \times(0, T)
$$

with the initial condition

$$
u(x, 0)=u_{0}(x), \text { a.e. } x \in \Omega,
$$

and the boundary condition

$$
u(x, t)=0 \text {, a.e. }(x, t) \in \partial \Omega \times \mathbb{R}_{+},
$$

under some hypotheses on the real functions $f, g$, the initial data $u_{0}$, the right hand side $r$, and on the domain $\Omega$, which are detailed below. Note that the case of Neumann boundary conditions on a part of the boundary or on the whole boundary, instead of (3), is interesting as well, and that it does not add specific difficulties to the present study. The standard mean curvature flow level set equation, which is obtained by setting $r=0$ and

$$
f(x)=g(x)=x, \forall x \in \mathbb{R}_{+}
$$

in (1), has numerous applications in science, engineering and technology, ranging from free boundary problems in material sciences and computational fluid dynamics to filtering and segmentation algorithms in image processing and computer vision. We refer to [28] for the original mean curvature flow level set equation, to $[1,3,5,23,30]$ for some generalisations in various frameworks; in image processing applications, equation (1-4), called the curvature filter, is generalised and used in adaptive image filtering [8], image segmentation by the geodesic active contours [5, 23] and the (generalised) subjective surfaces method $[29,9,24]$.

\footnotetext{
*Université Paris-Est, France, robert.eymard@univ-mlv.fr

†Slovak University of Technology, angela@math.sk

‡Slovak University of Technology, mikula@math.sk
} 
The analysis of numerical algorithms for solving (1-4) and related problems is a difficult task due to its nonlinear character and non-divergent form. In [10, 11, 12], the error estimates for geometric quantities like the regularised normal to the level set of solution and its normal velocity have been established using the finite element method. Such estimates are very useful for the free boundary problems when dealing with the motion of one particular level curve or level surface.

On the other hand, e.g. in image processing applications when the evolution of the whole level set function representing an image intensity is used in practice, the convergence of a numerical approximation to the solution $u$ itself is an important point. Convergence of a specially designed finite difference scheme to the viscosity solution of (1-4) is given in [27]. The finite volume schemes may sometimes be preferred because of the piecewise constant representation of numerical solution [25] and due to natural $L^{\infty}$ - stability of the numerical schemes. A finite volume scheme is proposed by Walkington in [31]. It is based on the so-called co-volume strategy for solving (1), setting

$$
f(x)=g(x)=\sqrt{x^{2}+a^{2}}, \forall x \in \mathbb{R}_{+},
$$

for a small value $a>0$. This regularisation is used to prevent from the occurrence of zero denominators in numerical schemes. This regularisation (5), known as the Evans-Spruck regularisation of the problem, is used in $[15,7]$ to show the existence of the viscosity solution to (1-4). Walkington's scheme is nonlinear and its linear semi-implicit variant is suggested in [22]. Such semi-implicit scheme is proved to be efficient, as keeping all theoretical properties of Walkington's scheme. It is used in solving various practical 2D and 3D (large-scale) image analysis problems [9, 13, 24]. Theoretical properties of the semi-implicit co-volume scheme for solving such a regularisation of (4) are studied in [22, 26] and [21]. In [22, 26] the $L^{\infty}$ stability of solution and $L^{1}$ stability of solution gradient are given and, moreover, in [21], the consistency of the scheme is proved using the Barles and Souganidis [4] approach for solving nonlinear PDEs. However, the convergence of the co-volume semi-implicit scheme to the exact solution remains an open problem.

Note that the convergence of finite volume methods for the solution of the stationary version of (1), has been proven in $[2,14,19]$, under the assumptions

(LL1) the function $x \mapsto x / f(x)$ is strictly increasing on $\mathbb{R}_{+}$,

$(\mathrm{LL} 2) \frac{d x}{c+x^{p-1}} \leq f(x) \leq C x^{2-p}$ for given $c, d, C>0, p>1$ and all $x \in \mathbb{R}_{+}$,

(LL3) $g$ constant.

We get under assumptions (LL1)-(LL2) that the function $u \mapsto-\operatorname{div}(\nabla u / f(|\nabla u|))$ is a Leray-Lions operator, whose monotony properties allow for the use of Minty and Leray-Lions tricks for the proof of the convergence. Note that property (LL1) holds for the choice (5) for $f$, but not (LL2). On the contrary, (LL1)-(LL2) hold if we consider for example

$$
f(x)=g(x)=\min \left(\sqrt{x^{2}+a^{2}}, b\right), \forall x \in \mathbb{R}_{+},
$$

for given reals $0<a \leq b$, setting $p=2, c=1, d=a$ and $C=b$. In the choice (6), the use of the bound $b$ is in accordance with image processing applications. Indeed, on discrete grids, the gradient norms are lower than $\frac{Q}{h}$, where $Q$ is a quantisation parameter and $h$ is the side length of a pixel. This acts in a similar way to the convolution used in [6] for regularising the Perona-Malik equation. Nevertheless, the problem approximated in $[2,14,19]$ is stationary and conservative; new difficulties arise in approximating (1), which is evolutive and nonconservative. In order to be able to overcome these difficulties, we consider in this paper the following hypotheses, called hypotheses $(\mathrm{H})$ in the following.

1. $\Omega$ is a finite connected open subset of $\mathbb{R}^{d}, d \in \mathbb{N}^{\star}$, with boundary $\partial \Omega$ defined by a finite union of subsets of hyperplanes of $\mathbb{R}^{d}$,

2. $u_{0} \in H_{0}^{1}(\Omega)$,

3. $r \in L^{2}(\Omega \times(0, T))$ for all $T>0$, 
4. $g \in C^{0}\left(\mathbb{R}_{+} ;[a, b]\right)$, with $0<a<b$,

5. $f \in C^{0}\left(\mathbb{R}_{+} ;[a, b]\right)$ is a Lipschitz continuous (non-strictly) increasing function, such that the function $x \mapsto x / f(x)$ is strictly increasing on $\mathbb{R}_{+}$.

It is worth noticing that the functions $f$ and $g$ given by (6) satisfy (H4-5).

Definition 1.1 (Weak solution of (1)-(2)-(3)) Under hypotheses (H), we say that $u$ is a weak solution of (1)-(2)-(3) if, for all $T>0$,

1. $u \in L^{2}\left(0, T ; H_{0}^{1}(\Omega)\right)$ and $u_{t} \in L^{2}(\Omega \times(0, T))$ (hence $\left.u \in C^{0}\left(0, T ; L^{2}(\Omega)\right)\right)$.

2. $u(\cdot, 0)=u_{0}$

3. the following holds

$$
\begin{aligned}
& \int_{0}^{T} \int_{\Omega}\left(\frac{u_{t}(x, t) v(x, t)}{g(|\nabla u(x, t)|)}+\frac{\nabla u(x, t) \cdot \nabla v(x, t)}{f(|\nabla u(x, t)|)}\right) \mathrm{d} x \mathrm{~d} t=\int_{0}^{T} \int_{\Omega} \frac{r(x, t) v(x, t)}{g(|\nabla u(x, t)|)} \mathrm{d} x \mathrm{~d} t \\
& \forall v \in L^{2}\left(0, T ; H_{0}^{1}(\Omega)\right) .
\end{aligned}
$$

Since any function $u$ weak solution of (1)-(2)-(3) in the sense of Definition 1.1 satisfies div $\left(\frac{\nabla u}{f(|\nabla u|)}\right) \in$ $L^{2}(\Omega \times(0, T))$, we immediately get the following lemma.

Lemma 1.1 (Property of weak solutions of (1)-(2)-(3)) Under Hypotheses (H), $u$ is a weak solution of (1)-(2)-(3) in the sense of Definition 1.1 if and only if $u$ satisfies, for all $T>0$ :

1. $u \in L^{2}\left(0, T ; H_{0}^{1}(\Omega)\right)$ and $u_{t} \in L^{2}(\Omega \times(0, T))$ (hence $\left.u \in C^{0}\left(0, T ; L^{2}(\Omega)\right)\right)$,

2. $u(\cdot, 0)=u_{0}$,

3. $\operatorname{div}\left(\frac{\nabla u}{f(|\nabla u|)}\right) \in L^{2}(\Omega \times(0, T))$,

4. $u_{t}-g(|\nabla u|) \operatorname{div}\left(\frac{\nabla u}{f(|\nabla u|)}\right)=r$ a.e. in $\Omega \times(0, T)$.

Remark 1.1 The framework of this paper does not easily allow for using uniqueness results, deduced from the viscosity solution sense. Difficulties arise, when considering general functions $f$ and $g$, initial data only in $H_{0}^{1}(\Omega)$, Lipschitz-continuous boundary for Dirichlet boundary condition. Moreover, the monotony of the problem has to be checked, and the relation between a solution in the viscosity sense and the sense of Definition 1.1 is not straightforward. Hence the uniqueness of the weak solution of (1)-(2)-(3) in the sense of Definition 1.1 is at this time an open problem.

We consider in this paper two different time discretisations of a new finite volume scheme for solving (1) under Hypotheses $(\mathrm{H})$. The main result of this paper, i.e. the strong convergence of both schemes to a solution of (7), is proven thanks to the following property. Let $F$ be the function defined by

$$
\forall s \in \mathbb{R}_{+}, F(s)=\int_{0}^{s} \frac{z}{f(z)} \mathrm{d} z \in\left[\frac{s^{2}}{2 b}, \frac{s^{2}}{2 a}\right] .
$$

Then, for any sufficiently regular function $u$, it holds

$$
\frac{\mathrm{d}}{\mathrm{d} t} \int_{\Omega} F(|\nabla u(x, t)|) \mathrm{d} x=\int_{\Omega} \frac{\nabla u(x, t) \cdot \nabla u_{t}(x, t)}{f(|\nabla u(x, t)|)} \mathrm{d} x \mathrm{~d} t .
$$

Therefore, assuming that this function $u$ is solution of (1) with $r=0$ for the sake of simplicity, we get, by taking $v=u_{t}$ in $(7)$, that $\nabla u \in C^{0}\left([0, T] ; L^{2}(\Omega)\right)$ and

$$
\int_{0}^{T} \int_{\Omega}\left(\frac{u_{t}(x, t)^{2}}{g(|\nabla u(x, t)|)}\right) \mathrm{d} x \mathrm{~d} t+\int_{\Omega} F(|\nabla u(x, T)|) \mathrm{d} x=\int_{\Omega} F\left(\left|\nabla u_{0}(x)\right|\right) \mathrm{d} x .
$$


The discrete equivalent of this property is shown in Lemma 3.2 for the semi implicit scheme (using the fact that $f$ is increasing). The hypothesis that $x \mapsto x / f(x)$ is strictly increasing is used by Minty and Leray-Lions tricks; unfortunately, although it is possible to extend some of these properties to the case $f(x)=x$, the convergence study provided in this paper does not hold in this framework, nor Lemma 1.1.

This paper is organised as follows. In Section 2, we present the discretisation tools. Then in Section (3), we show some estimates that are crucial in the convergence proof, given in Section (4). Finally, numerical results are given in Section (5), before an appendix containing a few classical technical results.

\section{The finite volume schemes}

In order to describe the schemes, we now introduce some notations for the space discretisation.

Definition 2.1 (Space discretisation) Let $\Omega$ be a polyhedral open bounded connected subset of $\mathbb{R}^{d}$, with $d \in \mathbb{N} \backslash\{0\}$, and $\partial \Omega=\bar{\Omega} \backslash \Omega$ its boundary. A discretisation of $\Omega$, denoted by $\mathcal{D}$, is defined as the triplet $\mathcal{D}=(\mathcal{M}, \mathcal{E}, \mathcal{P})$, where:

1. $\mathcal{M}$ is a finite family of nonempty connected open disjoint subsets of $\Omega$ (the "control volumes") such that $\bar{\Omega}=\cup_{p \in \mathcal{M}} \bar{p}$. For any $p \in \mathcal{M}$, let $\partial p=\bar{p} \backslash p$ be the boundary of $p$; let $|p|>0$ denote the measure of $p$ and let $h_{p}$ denote the diameter of $p$ and $h_{\mathcal{D}}$ denote the maximum value of $\left(h_{p}\right)_{m \in \mathcal{M}}$.

2. $\mathcal{E}$ is a finite family of disjoint subsets of $\bar{\Omega}$ (the "edges" of the mesh), such that, for all $\sigma \in \mathcal{E}, \sigma$ is a nonempty open subset of a hyperplane of $\mathbb{R}^{d}$, whose $(d-1)$-dimensional measure $|\sigma|$ is strictly positive. We also assume that, for all $p \in \mathcal{M}$, there exists a subset $\mathcal{E}_{p}$ of $\mathcal{E}$ such that $\partial p=\cup_{\sigma \in \mathcal{E}_{p}} \bar{\sigma}$. For any $\sigma \in \mathcal{E}$, we denote by $\mathcal{M}_{\sigma}=\left\{p \in \mathcal{M}, \sigma \in \mathcal{E}_{p}\right\}$. We then assume that, for all $\sigma \in \mathcal{E}$, either $\mathcal{M}_{\sigma}$ has exactly one element and then $\sigma \subset \partial \Omega$ (the set of these interfaces, called boundary interfaces, is denoted by $\mathcal{E}_{\text {ext }}$ ) or $\mathcal{M}_{\sigma}$ has exactly two elements (the set of these interfaces, called interior interfaces, is denoted by $\left.\mathcal{E}_{\text {int }}\right)$. For all $\sigma \in \mathcal{E}$, we denote by $x_{\sigma}$ the barycentre of $\sigma$. For all $p \in \mathcal{M}$ and $\sigma \in \mathcal{E}_{p}$, we denote by $\mathbf{n}_{p, \sigma}$ the unit vector normal to $\sigma$ outward to $p$.

3. $\mathcal{P}$ is a family of points of $\Omega$ indexed by $\mathcal{M}$, denoted by $\mathcal{P}=\left(x_{p}\right)_{p \in \mathcal{M}}$, such that for all $p \in \mathcal{M}, x_{p} \in p$ and $p$ is assumed to be $x_{p}$-star-shaped, which means that for all $x \in p$, the inclusion $\left[x_{p}, x\right] \subset p$ holds. Denoting by $d_{p \sigma}$ the Euclidean distance between $x_{p}$ and the hyperplane including $\sigma$, one assumes that $d_{p \sigma}>0$. We then denote by $D_{p, \sigma}$ the cone with vertex $x_{p}$ and basis $\sigma$.

4. We make the important following assumption:

$$
d_{p \sigma} \mathbf{n}_{p, \sigma}=x_{\sigma}-x_{p}, \forall p \in \mathcal{M}, \forall \sigma \in \mathcal{E}_{p}
$$

Remark 2.1 The preceding definition applies to triangular meshes if $d=2$, with all angles acute, and to meshes build with orthogonal parallelepipedic control volumes (rectangles if $d=2$ ).

We denote

$$
\theta_{\mathcal{D}}=\min _{p \in \mathcal{M}} \min _{\sigma \in \mathcal{E}_{p}} \frac{d_{p \sigma}}{h_{p}} .
$$

Definition 2.2 (Space-time discretisation) Let $\Omega$ be a polyhedral open bounded connected subset of $\mathbb{R}^{d}$, with $d \in \mathbb{N}^{\star}$ (where $\mathbb{N}^{\star}$ denotes the set $\mathbb{N} \backslash\{0\}$ ) and let $T>0$ be given. We say that $(\mathcal{D}, \tau)$ is a space-time discretisation of $\Omega \times(0, T)$ if $\mathcal{D}$ is a space discretisation of $\Omega$ in the sense of Definition 2.1 and if there exists $N_{T} \in \mathbb{N}$ with $T=\left(N_{T}+1\right) \tau$.

Let $(\mathcal{D}, \tau)$ be a space-time discretisation of $\Omega \times(0, T)$. We define the set $H_{\mathcal{D}} \subset \mathbb{R}^{\mathcal{M}} \times \mathbb{R}^{\mathcal{E}}$ such that $u_{\sigma}=0$ for all $\sigma \in \mathcal{E}_{\text {ext }}$. We define the following functions on $H_{\mathcal{D}}$ :

$$
N_{p}(u)^{2}=\frac{1}{|p|} \sum_{\sigma \in \mathcal{E}_{p}} \frac{|\sigma|}{d_{p \sigma}}\left(u_{\sigma}-u_{p}\right)^{2}, \forall p \in \mathcal{M}, \forall u \in H_{\mathcal{D}} .
$$


Let us recall that

$$
\|u\|_{1, \mathcal{D}}^{2}=\sum_{p \in \mathcal{M}}|p| N_{p}(u)^{2}
$$

defines a norm on $H_{\mathcal{D}}$ (see [20]). We then define the set $H_{\mathcal{D}, \tau}$ of all $u=\left(u^{n+1}\right)_{n=0, \ldots, N_{T}}$ such that $u^{n+1} \in H_{\mathcal{D}}$ for all $n=0, \ldots, N_{T}$, and we set

$$
\|u\|_{1, \mathcal{D}, \tau}^{2}=\sum_{n=0}^{N_{T}} \tau\left\|u^{n+1}\right\|_{1, \mathcal{D}}^{2}, \forall u \in H_{\mathcal{D}, \tau}
$$

We now define two numerical schemes. The fully implicit scheme is defined by

$$
\begin{gathered}
u_{p}^{0}=\frac{1}{|p|} \int_{p} u_{0}(x) \mathrm{d} x, \forall p \in \mathcal{M}, \\
r_{p}^{n+1}=\int_{n \tau}^{(n+1) \tau} \int_{p} r(x, t) \mathrm{d} x \mathrm{~d} t, \forall p \in \mathcal{M}, \forall n \in \mathbb{N},
\end{gathered}
$$

and

$$
\begin{aligned}
& \frac{|p|}{\tau g\left(N_{p}\left(u^{n+1}\right)\right)}\left(u_{p}^{n+1}-u_{p}^{n}\right)-\frac{1}{f\left(N_{p}\left(u^{n+1}\right)\right)} \sum_{\sigma \in \mathcal{E}_{p}} \frac{|\sigma|}{d_{p \sigma}}\left(u_{\sigma}^{n+1}-u_{p}^{n+1}\right)=\frac{r_{p}^{n+1}}{\tau g\left(N_{p}\left(u^{n+1}\right)\right)} \\
& \forall p \in \mathcal{M}, \forall n \in \mathbb{N}
\end{aligned}
$$

the following relation is given for the interior edges,

$$
\frac{u_{\sigma}^{n+1}-u_{p}^{n+1}}{f\left(N_{p}\left(u^{n+1}\right)\right) d_{p \sigma}}+\frac{u_{\sigma}^{n+1}-u_{q}^{n+1}}{f\left(N_{q}\left(u^{n+1}\right)\right) d_{q \sigma}}=0, \forall \sigma \in \mathcal{E}_{\text {int }} \text { with } \mathcal{M}_{\sigma}=\{p, q\}, \forall n \in \mathbb{N},
$$

and the boundary condition is fulfilled thanks to

$$
u_{\sigma}^{n+1}=0, \forall \sigma \in \mathcal{E}_{\text {ext }}, \forall n \in \mathbb{N} .
$$

The semi-implicit scheme is defined by (16),

$$
u_{\sigma}^{0}=\frac{1}{|\sigma|} \int_{\sigma} u_{0}(x) \mathrm{d} s(x), \forall \sigma \in \mathcal{E},
$$

(17), (20) and

$$
\begin{aligned}
& \frac{|p|}{\tau g\left(N_{p}\left(u^{n}\right)\right)}\left(u_{p}^{n+1}-u_{p}^{n}\right)-\frac{1}{f\left(N_{p}\left(u^{n}\right)\right)} \sum_{\sigma \in \mathcal{E}_{p}} \frac{|\sigma|}{d_{p \sigma}}\left(u_{\sigma}^{n+1}-u_{p}^{n+1}\right)=\frac{r_{p}^{n+1}}{\tau g\left(N_{p}\left(u^{n}\right)\right)}, \\
& \forall p \in \mathcal{M}, \forall n \in \mathbb{N}
\end{aligned}
$$

where the following relation is given for the interior edges

$$
\frac{u_{\sigma}^{n+1}-u_{p}^{n+1}}{f\left(N_{p}\left(u^{n}\right)\right) d_{p \sigma}}+\frac{u_{\sigma}^{n+1}-u_{q}^{n+1}}{f\left(N_{q}\left(u^{n}\right)\right) d_{q \sigma}}=0, \forall \sigma \in \mathcal{E}_{\text {int }} \text { with } \mathcal{M}_{\sigma}=\{p, q\}, \forall n \in \mathbb{N}
$$

In the following, for the sake of shortness and clarity, all properties concerning the fully implicit scheme will be only sketched in remarks, focusing on the semi-implicit scheme. Hence, now considering a family of values $\left(u_{p}^{n}\right)_{p \in \mathcal{M}, n \in \mathbb{N}}$, given by (16), (17), (20) and (21), (22), (23), we define the approximate solution $u_{\mathcal{D}, \tau}$ in $\Omega \times \mathbb{R}_{+}$by

$$
\left.\left.u_{\mathcal{D}, \tau}(x, 0)=u_{p}^{0}, u_{\mathcal{D}, \tau}(x, t)=u_{p}^{n+1} \text {, for a.e. } x \in p, \forall t \in\right] n \tau,(n+1) \tau\right], \forall p \in \mathcal{M}, \forall n \in \mathbb{N} .
$$


We then define $w_{\mathcal{D}, \tau}, z_{\mathcal{D}, \tau}, N_{\mathcal{D}, \tau}$ and $\widetilde{N}_{\mathcal{D}, \tau}$ by

$$
\begin{aligned}
& w_{\mathcal{D}, \tau}(x, t)=-\frac{u_{p}^{n+1}-u_{p}^{n}}{\tau g\left(N_{p}\left(u^{n}\right)\right)}+\frac{r_{p}^{n+1}}{|p| \tau g\left(N_{p}\left(u^{n}\right)\right)}, \quad z_{\mathcal{D}, \tau}(x, t)=\frac{u_{p}^{n+1}-u_{p}^{n}}{\tau}, \\
& N_{\mathcal{D}, \tau}(x, t)=N_{p}\left(u^{n+1}\right), \widetilde{N}_{\mathcal{D}, \tau}(x, t)=N_{p}\left(u^{n}\right), \\
& \text { for a.e. } x \in p, \quad \text { for a.e. } t \in] n \tau,(n+1) \tau[, \quad \forall p \in \mathcal{M}, \quad \forall n \in \mathbb{N} .
\end{aligned}
$$

Finally, we define $G_{\mathcal{D}, \tau}, H_{\mathcal{D}, \tau}$ and $\widetilde{H}_{\mathcal{D}, \tau}$ by

$$
\begin{aligned}
& G_{\mathcal{D}, \tau}(x, t)=d \frac{u_{\sigma}^{n+1}-u_{p}^{n+1}}{d_{p \sigma}} \mathbf{n}_{p \sigma}, \\
& H_{\mathcal{D}, \tau}(x, t)=d \frac{u_{\sigma}^{n+1}-u_{p}^{n+1}}{d_{p \sigma} f\left(N_{p}\left(u^{n+1}\right)\right)} \mathbf{n}_{p \sigma}, \quad \widetilde{H}_{\mathcal{D}, \tau}(x, t)=d \frac{u_{\sigma}^{n+1}-u_{p}^{n+1}}{d_{p \sigma} f\left(N_{p}\left(u^{n}\right)\right)} \mathbf{n}_{p \sigma}, \\
&\text { for a.e. } \left.x \in D_{p \sigma}, \quad \text { for a.e. } t \in\right] n \tau,(n+1) \tau\left[, \forall p \in \mathcal{M}, \forall \sigma \in \mathcal{E}_{p}, \forall n \in \mathbb{N},\right.
\end{aligned}
$$

(recall that $D_{p \sigma}$ is the cone with vertex $x_{p}$ and basis $\sigma$ and $\mathbf{n}_{p \sigma}$ is the normal unit vector to $\sigma$ outward to $p$ ). Note that $u_{\mathcal{D}, \tau}$ is the solution of

$$
\begin{gathered}
-\frac{1}{f\left(N_{p}\left(u^{n}\right)\right)} \sum_{\sigma \in \mathcal{E}_{p}} \frac{|\sigma|}{d_{p \sigma}}\left(u_{\sigma}^{n+1}-u_{p}^{n+1}\right)=|p| w_{p}^{n+1}, \forall p \in \mathcal{M}, \forall n \in \mathbb{N}, \\
\frac{u_{\sigma}^{n+1}-u_{p}^{n+1}}{f\left(N_{p}\left(u^{n}\right)\right) d_{p \sigma}}+\frac{u_{\sigma}^{n+1}-u_{q}^{n+1}}{f\left(N_{q}\left(u^{n}\right)\right) d_{q \sigma}}=0, \forall \sigma=p \mid q \in \mathcal{E}_{\mathrm{int}}, \quad \forall n \in \mathbb{N},
\end{gathered}
$$

and

$$
u_{\sigma}^{n+1}=0, \forall \sigma \in \mathcal{E}_{\text {ext }}, \forall n \in \mathbb{N} .
$$

The next section is devoted to the study of some estimates satisfies by the discrete solution. These estimates in particular allow for the proof of the existence and uniqueness of the discrete solution. These estimates also give rise to a brief review of a few properties in the case of Crank-Nicolson versions of these schemes, which are confirmed by the numerical tests shown in section 5 .

\section{Estimates}

Let us now state the $L^{\infty}$ stability of the scheme.

Lemma 3.1 ( $L^{\infty}$ stability of the scheme, existence and uniqueness of the discrete solution) Under Hypotheses $(H)$, let $(\mathcal{D}, \tau)$ be a space-time discretisation of $\Omega \times(0, T)$ in the sense of Definition 2.2. We denote by

$$
\left|u_{0}\right|_{\mathcal{D}, \infty}=\max _{p \in \mathcal{M}}\left|u_{p}^{0}\right|
$$

and by

$$
|r|_{\mathcal{D}, \tau, \infty}=\max \left\{\frac{\left|r_{p}^{n+1}\right|}{\tau|p|}, p \in \mathcal{M}, n=0, \ldots, N_{T}\right\}
$$

(note that, if $u_{0} \in L^{\infty}(\Omega)$ and $r \in L^{\infty}\left(\Omega \times \mathbb{R}_{+}\right)$, then $\left|u_{0}\right|_{\mathcal{D}, \infty} \leq\left\|u_{0}\right\|_{L^{\infty}(\Omega)}$ and $\left.|r|_{\mathcal{D}, \tau, \infty} \leq\|r\|_{L^{\infty}(\Omega \times(0, T))}\right)$. Let $\left(u_{p}^{n}\right)_{p \in \mathcal{M}, n \in \mathbb{N}}$ be a solution of (16), (17), (20) and (21), (22), (23). Then it holds:

$$
\left|u_{p}^{n}\right| \leq\left|u_{0}\right|_{\mathcal{D}, \infty}+|r|_{\mathcal{D}, \tau, \infty} n \tau \leq\left|u_{0}\right|_{\mathcal{D}, \infty}+|r|_{\mathcal{D}, \tau, \infty} T, \forall p \in \mathcal{M}, \forall n=0, \ldots, N_{T} .
$$

As a straightforward consequence, there exists one and only one solution to the semi-implicit scheme (21), (17), (20), (22), (23). 
Proof. Suppose that for fixed time step $(n+1)$ the maximum of all $u_{p}^{n+1}$ is achieved at the finite volume $p$. Let us write (22) in the following way:

$$
u_{p}^{n+1}+\frac{\tau g\left(N_{p}\left(u^{n}\right)\right)}{|p| f\left(N_{p}\left(u^{n}\right)\right)} \sum_{\sigma \in \mathcal{E}_{p}} \frac{|\sigma|}{d_{p \sigma}}\left(u_{p}^{n+1}-u_{\sigma}^{n+1}\right)=u_{p}^{n}+\frac{r_{p}^{n+1}}{|p|} .
$$

Since the value $u_{\sigma}^{n+1}$ satisfies the equality

$$
u_{\sigma}^{n+1}=\frac{u_{p}^{n+1} f\left(N_{q}\left(u^{n}\right)\right) d_{q \sigma}+u_{q}^{n+1} f\left(N_{p}\left(u^{n}\right)\right) d_{p \sigma}}{f\left(N_{p}\left(u^{n}\right)\right) d_{p \sigma}+f\left(N_{q}\left(u^{n}\right)\right) d_{q \sigma}},
$$

which is a convex linear combination of points $u_{p}^{n+1}, u_{q}^{n+1}$, we obtain

$$
u_{p}^{n+1}-u_{\sigma}^{n+1}=\frac{f\left(N_{p}\left(u^{n}\right)\right) d_{p \sigma}\left(u_{p}^{n+1}-u_{q}^{n+1}\right)}{f\left(N_{p}\left(u^{n}\right)\right) d_{p \sigma}+f\left(N_{q}\left(u^{n}\right)\right) d_{q \sigma}},
$$

which is nonnegative. This leads to

$$
u_{p}^{n+1} \leq u_{p}^{n}+|r|_{\mathcal{D}, \tau, \infty} \tau .
$$

Then, we recursively get the estimate (34), similarly reasoning for the minimum values.

Remark 3.1 The above proof also applies for the fully implicit scheme, only replacing $n$ by $n+1$ in the arguments of functions $f$ and $g$, allowing for a proof of existence of at least one discrete solution, thanks to Brouwer's fixed point theorem. Note that the uniqueness is not proved, as in the continuous case.

Lemma 3.2 $L^{2}(\Omega \times(0, T))$ estimate on $u_{t}$ and $L^{\infty}\left(0, T ; H_{\mathcal{D}}\right)$ estimate. Let Hypotheses $(H)$ be fulfilled. Let $(\mathcal{D}, \tau)$ be a space-time discretisation of $\Omega \times(0, T)$ in the sense of Definition 2.2 and let $\theta \in] 0, \theta_{\mathcal{D}}\left[\right.$, where $\theta_{\mathcal{D}}$ is defined by (12). Let $\left(u_{p}^{n}\right)_{p \in \mathcal{M}, n \in \mathbb{N}}$ be the solution of (16), (17), (20) and (21), (22), (23). Then there exists $C_{\theta}>0$, only depending on $\theta$, such that it holds:

$$
\begin{aligned}
& \frac{1}{2 b} \sum_{n=0}^{m-1} \tau \sum_{p \in \mathcal{M}}|p|\left(\frac{u_{p}^{n+1}-u_{p}^{n}}{\tau}\right)^{2}+\sum_{p \in \mathcal{M}}|p| F\left(N_{p}\left(u^{m}\right)\right) \\
& +\frac{1}{2 b} \sum_{n=0}^{m-1} \sum_{p \in \mathcal{M}}|p|\left(N_{p}\left(u^{n+1}\right)-N_{p}\left(u^{n}\right)\right)^{2} \leq \frac{C_{\theta}\left\|u^{0}\right\|_{H^{1}(\Omega)}^{2}+\|r\|_{L^{2}(\Omega \times(0, T))}^{2}}{2 a}, \forall m=1, \ldots, N_{T} .
\end{aligned}
$$

Proof. We multiply the scheme by $u_{p}^{n+1}-u_{p}^{n}$ and sum over $p$. We obtain $T_{1}+T_{2}=T_{3}$, where

$$
\begin{gathered}
T_{1}=\sum_{p \in \mathcal{M}} \frac{|p|}{\tau g\left(N_{p}\left(u^{n}\right)\right)}\left(u_{p}^{n+1}-u_{p}^{n}\right)^{2}, \\
T_{2}=\sum_{p \in \mathcal{M}} \frac{1}{f\left(N_{p}\left(u^{n}\right)\right)} \sum_{\sigma \in \mathcal{E}_{p}} \frac{|\sigma|}{d_{p \sigma}}\left(u_{\sigma}^{n+1}-u_{p}^{n+1}\right)\left(u_{\sigma}^{n+1}-u_{p}^{n+1}-\left(u_{\sigma}^{n}-u_{p}^{n}\right)\right), \\
T_{3}=\sum_{p \in \mathcal{M}} \frac{r_{p}^{n+1}}{\tau g\left(N_{p}\left(u^{n}\right)\right)}\left(u_{p}^{n+1}-u_{p}^{n}\right)
\end{gathered}
$$

where we have used the properties of the finite volume scheme. We first remark that, thanks to Young's inequality and to the Cauchy-Schwarz inequality

$$
T_{3} \leq \sum_{p \in \mathcal{M}} \frac{\left(r_{p}^{n+1}\right)^{2}}{2|p| \tau g\left(N_{p}\left(u^{n}\right)\right)}+\frac{1}{2} T_{1} \leq \frac{1}{2 a} \int_{n \tau}^{(n+1) \tau} \int_{\Omega} r(x, t)^{2} \mathrm{~d} x \mathrm{~d} t+\frac{1}{2} T_{1} .
$$


Let us turn to the study of $T_{2}$. Using Definition (8) of function $F$, we have:

$$
F\left(N_{p}\left(u^{n+1}\right)\right)-F\left(N_{p}\left(u^{n}\right)\right)=\int_{N_{p}\left(u^{n}\right)}^{N_{p}\left(u^{n+1}\right)} \frac{z \mathrm{~d} z}{f(z)}
$$

We remark that, thanks to Hypothesis (H5),

$$
\forall c, d \in \mathbb{R}_{+}, \int_{c}^{d} \frac{z \mathrm{~d} z}{f(z)}+\frac{(d-c)^{2}}{2 f(c)} \leq \frac{d}{f(c)}(d-c) .
$$

Indeed, we set, for $c, d \in \mathbb{R}_{+}, \Phi_{c}(d)=\frac{d}{f(c)}(d-c)-\frac{(d-c)^{2}}{2 f(c)}-\int_{c}^{d} \frac{z \mathrm{~d} z}{f(z)}$. We have $\Phi_{c}(c)=0$, and $\Phi_{c}^{\prime}(d)=$ $\frac{d}{f(c)}-\frac{d}{f(d)}$, whose sign is that of $d-c$ since $f$ is (non-strictly) increasing. Hence $\Phi_{c}(d) \geq 0$ and we get

$$
F\left(N_{p}\left(u^{n+1}\right)\right)-F\left(N_{p}\left(u^{n}\right)\right)+\frac{1}{2 b}\left(N_{p}\left(u^{n+1}\right)-N_{p}\left(u^{n}\right)\right)^{2} \leq \frac{N_{p}\left(u^{n+1}\right)}{f\left(N_{p}\left(u^{n}\right)\right)}\left(N_{p}\left(u^{n+1}\right)-N_{p}\left(u^{n}\right)\right) .
$$

Note that the Cauchy-Schwarz inequality implies

$$
\sum_{\sigma \in \mathcal{E}_{p}} \frac{|\sigma|}{d_{p \sigma}}\left(u_{\sigma}^{n+1}-u_{p}^{n+1}\right)\left(u_{\sigma}^{n}-u_{p}^{n}\right) \leq|p| \quad N_{p}\left(u^{n}\right) N_{p}\left(u^{n+1}\right),
$$

which in turn implies

$$
\sum_{p \in \mathcal{M}}|p|\left(F\left(N_{p}\left(u^{n+1}\right)\right)-F\left(N_{p}\left(u^{n}\right)\right)+\frac{1}{2 b} \sum_{p \in \mathcal{M}}|p|\left(N_{p}\left(u^{n+1}\right)-N_{p}\left(u^{n}\right)\right)^{2} \leq T_{2} .\right.
$$

Finally we obtain

$$
\begin{aligned}
& \frac{1}{2 b} \tau \sum_{p \in \mathcal{M}}|p|\left(\frac{u_{p}^{n+1}-u_{p}^{n}}{\tau}\right)^{2}+\sum_{p \in \mathcal{M}}|p| F\left(N_{p}\left(u^{n+1}\right)\right)+\frac{1}{2 b} \sum_{p \in \mathcal{M}}|p|\left(N_{p}\left(u^{n+1}\right)-N_{p}\left(u^{n}\right)\right)^{2} \\
& \leq \sum_{p \in \mathcal{M}}|p| F\left(N_{p}\left(u^{n}\right)\right)+\frac{1}{2 a} \int_{n \tau}^{(n+1) \tau} \int_{\Omega} r(x, t)^{2} \mathrm{~d} x \mathrm{~d} t,
\end{aligned}
$$

and summing this inequality over $n=0, \ldots, m-1$ for all $m=1, \ldots, N_{T}$, we get that

$$
\begin{aligned}
& \frac{1}{2 b} \sum_{n=0}^{m-1} \tau \sum_{p \in \mathcal{M}}|p|\left(\frac{u_{p}^{n+1}-u_{p}^{n}}{\tau}\right)^{2}+\sum_{p \in \mathcal{M}}|p| F\left(N_{p}\left(u^{m}\right)\right) \\
& +\frac{1}{2 b} \sum_{n=0}^{m-1} \sum_{p \in \mathcal{M}}|p|\left(N_{p}\left(u^{n+1}\right)-N_{p}\left(u^{n}\right)\right)^{2} \\
& \leq \sum_{p \in \mathcal{M}}|p| F\left(N_{p}\left(u^{0}\right)\right)+\frac{1}{2 a} \int_{0}^{m \tau} \int_{\Omega} r(x, t)^{2} \mathrm{~d} x \mathrm{~d} t,
\end{aligned}
$$

where we define $u_{\sigma}^{0}$ by (21). We then use the following inequality, proven in [18]: there exists $C_{\theta}>0$, only depending on $\theta$, such that

$$
|p| N_{p}\left(u^{0}\right)^{2} \leq C_{\theta}\left\|u_{0}\right\|_{H^{1}(p)}^{2}, \forall p \in \mathcal{M}
$$

We thus get (35). 
Remark 3.2 A property, similar to that stated in Lemma 3.2, can be shown for the fully implicit scheme. One should remark that, in this case, there is no term in $\left(N_{p}\left(u^{n+1}\right)-N_{p}\left(u^{n}\right)\right)^{2}$ in the discrete relation issued from the computations, which rely on the monotony of the function $x \mapsto x / f(x)$ and not on that of $f$. This estimate then also allows for proving the existence of at least one solution to the fully implicit scheme, using the topological degree argument.

Remark 3.3 (Case of the non-regularised level set equation) If we use the hypothesis $s / f(s) \leq a$ (which holds for $f(s)=s$ ) instead of $a \leq f(s)$, assumed in this paper, the above computations provide an $L^{\infty}\left(0, T ; L^{1}(\Omega)\right)$ estimate on the discrete gradient instead of an $L^{\infty}\left(0, T ; L^{2}(\Omega)\right)$ estimate. It would nevertheless be possible to get some of the results proven during the convergence study, but not all of them. This is not surprising, since for the level set equation, there is no weak/strong sense, and we should refer to the viscosity solution sense. Hence the convergence study for $f(s)=s$ remains open.

\section{Consequences on Crank-Nicolson -like versions of the schemes}

In this paper, we could as well, for a given $\alpha \in\left[\frac{1}{2}, 1\right]$, replace (22) and (23) by

$$
\begin{aligned}
& \frac{|p|}{\tau g\left(N_{p}\left(u^{n}\right)\right)}\left(u_{p}^{n+1}-u_{p}^{n}\right)-\frac{1}{f\left(N_{p}\left(u^{n}\right)\right)} \sum_{\sigma \in \mathcal{E}_{p}} \frac{|\sigma|}{d_{p \sigma}}\left(\widehat{u}_{\sigma}^{n+1}-\widehat{u}_{p}^{n+1}\right)=\frac{r_{p}^{n+1}}{\tau g\left(N_{p}\left(u^{n}\right)\right)}, \\
& \widehat{u}_{p}^{n+1}=\alpha u_{p}^{n+1}+(1-\alpha) u_{p}^{n}, \\
& \forall p \in \mathcal{M}, \forall n \in \mathbb{N},
\end{aligned}
$$

and

$$
\frac{\widehat{u}_{\sigma}^{n+1}-\widehat{u}_{p}^{n+1}}{f\left(N_{p}\left(u^{n}\right)\right) d_{p \sigma}}+\frac{\widehat{u}_{\sigma}^{n+1}-\widehat{u}_{q}^{n+1}}{f\left(N_{q}\left(u^{n}\right)\right) d_{q \sigma}}=0, \forall \sigma \in \mathcal{E}_{\text {int }} \text { with } \mathcal{M}_{\sigma}=\{p, q\}, \forall n \in \mathbb{N}
$$

We then define the so-called " $\alpha$-scheme" version of the above semi-implicit scheme, which provides the Crank-Nicolson scheme for $\alpha=\frac{1}{2}$ and (22), (23) for $\alpha=1$. The convergence properties proven in this paper for $\alpha=1$ can be immediately generalised to the case $\left.\alpha \in] \frac{1}{2}, 1\right]$ for the semi-implicit scheme, since the crucial property (36) is modified into

$$
\forall c, d \in \mathbb{R}_{+}, \int_{c}^{d} \frac{z \mathrm{~d} z}{f(z)}+\left(\alpha-\frac{1}{2}\right) \frac{(d-c)^{2}}{f(c)} \leq \frac{\alpha d+(1-\alpha) c}{f(c)}(d-c),
$$

which holds under the same hypothesis $f$ increasing.

On the contrary, if, for a given $\alpha \in\left[\frac{1}{2}, 1\right]$, we replace (18) and (19) by

$$
\begin{aligned}
& \frac{|p|}{\tau g\left(N_{p}\left(\widehat{u}^{n+1}\right)\right)}\left(u_{p}^{n+1}-u_{p}^{n}\right)-\frac{1}{f\left(N_{p}\left(\widehat{u}^{n+1}\right)\right)} \sum_{\sigma \in \mathcal{E}_{p}} \frac{|\sigma|}{d_{p \sigma}}\left(\widehat{u}_{\sigma}^{n+1}-\widehat{u}_{p}^{n+1}\right)=\frac{r_{p}^{n+1}}{\tau g\left(N_{p}\left(\widehat{u}^{n+1}\right)\right)}, \\
& \widehat{u}_{p}^{n+1}=\alpha u_{p}^{n+1}+(1-\alpha) u_{p}^{n} \\
& \forall p \in \mathcal{M}, \forall n \in \mathbb{N}
\end{aligned}
$$

and

$$
\frac{\widehat{u}_{\sigma}^{n+1}-\widehat{u}_{p}^{n+1}}{f\left(N_{p}\left(\widehat{u}^{n+1}\right)\right) d_{p \sigma}}+\frac{\widehat{u}_{\sigma}^{n+1}-\widehat{u}_{q}^{n+1}}{f\left(N_{q}\left(\widehat{u}^{n+1}\right)\right) d_{q \sigma}}=0, \forall \sigma \in \mathcal{E}_{\text {int }} \text { with } \mathcal{M}_{\sigma}=\{p, q\}, \forall n \in \mathbb{N},
$$

we have to replace the fact that the function $s \mapsto s / f(s)$ is increasing by

$$
\forall c, d \in \mathbb{R}_{+}, \int_{c}^{d} \frac{z \mathrm{~d} z}{f(z)} \leq \frac{\alpha d+(1-\alpha) c}{f(\alpha d+(1-\alpha) c)}(d-c),
$$

which is not satisfied for all $\alpha \in\left[\frac{1}{2}, 1\right]$ by the example given in (6) (indeed, for $\alpha=\frac{1}{2}$, it implies that the function $s \mapsto s / f(s)$ is concave). 


\section{Convergence}

Thanks to the estimates proven in the above section, we are now in position for proving the convergence of the scheme, using the monotonicity properties of the operators. We first present a few properties which are useful in the convergence study. In this paper, we use the notations " $\rightarrow$ weakly" for denoting weak convergence and $\rightarrow$ for strong convergence.

Lemma 4.1 Let $\Omega$ be a bounded connected open subset of $\mathbb{R}^{d}$, with $d \in \mathbb{N}^{\star}$ and let $T>0$. Let $\left(\mathcal{D}_{m}, \tau_{m}\right)_{m \in \mathbb{N}}$ be a sequence of space-time discretisations of $\Omega$ in the sense of Definition 2.2 such that $h_{\mathcal{D}_{m}}$ tends to 0 as $m \longrightarrow \infty$. Let $\left(u_{m}\right)_{m \in \mathbb{N}}$ be such that $u_{m} \in H_{\mathcal{D}_{m}, \tau_{m}}$, such that $\left\|u_{m}\right\|_{1, \mathcal{D}_{m}, \tau_{m}} \leq C$ for all $m \in \mathbb{N}$ and such that there exists $\bar{u} \in L^{2}(\Omega \times(0, T))$ such that the sequence of functions $u_{\mathcal{D}_{m}, \tau_{m}}$ defined, for $u=u_{m}, \mathcal{D}=\mathcal{D}_{m}$ and $\tau=\tau_{m}$, by

$$
\left.\left.u_{\mathcal{D}, \tau}(x, t)=u_{p}^{n+1} \text {, for a.e. } x \in p, t \in\right] n \tau,(n+1) \tau\right], \forall p \in \mathcal{M}, \forall n=0, \ldots, N_{T},
$$

satisfies $u_{\mathcal{D}_{m}, \tau_{m}} \longrightarrow \bar{u}$ in $L^{2}(\Omega \times(0, T))$ as $m \longrightarrow \infty$.

Then $\bar{u} \in L^{2}\left(0, T ; H_{0}^{1}(\Omega)\right)$. Moreover, defining $G_{m} \in L^{\infty}\left(0, T ; L^{2}(\Omega)\right)$ by

$$
G_{m}(x, t)=d \frac{u_{\sigma}^{n+1}-u_{p}^{n+1}}{d_{p \sigma}} \mathbf{n}_{p \sigma}
$$

for a.e. $x \in D_{p \sigma}$, and a.e. $\left.t \in\right] n \tau,(n+1) \tau\left[\right.$, then $G_{m} \rightarrow \nabla \bar{u}$ weakly in $L^{2}(\Omega \times(0, T))^{d}$ as $m \longrightarrow \infty$.

Proof. We first notice that

$$
\left\|G_{m}\right\|_{L^{2}(\Omega \times(0, T))^{d}}^{2}=\sum_{n=0}^{N_{T}} \tau \sum_{p \in \mathcal{M}} \sum_{\sigma \in \mathcal{E}_{p}} \frac{|\sigma| d_{p \sigma}}{d}\left|d \frac{u_{\sigma}^{n+1}-u_{p}^{n+1}}{d_{p \sigma}} \mathbf{n}_{p \sigma}\right|^{2},
$$

which provides

$$
\left\|G_{m}\right\|_{L^{2}(\Omega \times(0, T))^{d}}^{2}=d\left(\left\|u_{m}\right\|_{1, \mathcal{D}_{m}, \tau_{m}}\right)^{2} .
$$

Prolonging $G_{m}$ (and $u_{m}$ ) by 0 outside $\Omega \times(0, T)$, we get that there exists $\bar{G} \in L^{2}\left(\mathbb{R}^{d} \times(0, T)\right)^{d}$ such that $G_{m} \rightarrow \bar{G}$ (weakly, up to a subsequence) in $L^{2}\left(\mathbb{R}^{d} \times(0, T)\right)^{d}$ as $m \longrightarrow \infty$. Moreover, $\bar{G}(x, t)=0$ for a.e. $x \notin \Omega \times(0, T)$. Let $\psi \in C_{c}^{1}\left(\mathbb{R}^{d} \times(0, T)\right)^{d}$. Let us define, for $\mathcal{D}=\mathcal{D}_{m}$ and $\tau=\tau_{m}$,

$$
\psi_{\sigma}^{n+1}=\frac{1}{|\sigma| \tau} \int_{n \tau}^{(n+1) \tau} \int_{\sigma} \psi(x, t) \mathrm{d} s(x) \mathrm{d} t, \sigma \in \mathcal{E}, n=0, \ldots, N_{T},
$$

and $\psi_{m}$ by

$$
\psi_{m}(x, t)=\psi_{\sigma}^{n+1},
$$

for a.e. $x \in D_{p, \sigma}$, all $p \in \mathcal{M}, \sigma \in \mathcal{E}_{p}$, a.e. $\left.t \in\right] n \tau,(n+1) \tau\left[\right.$ and all $n=0, \ldots, N_{T}$. Thanks to the regularity properties of $\psi$, we get that $\psi_{m} \rightarrow \psi$ in $L^{\infty}\left(\mathbb{R}^{d} \times(0, T)\right)^{d}$ as $m \longrightarrow \infty$. Moreover, we have

$$
\int_{0}^{T} \int_{\mathbb{R}^{d}} G_{m}(x, t) \cdot \psi_{m}(x, t) \mathrm{d} x \mathrm{~d} t=\sum_{n=0}^{N_{T}} \tau \sum_{p \in \mathcal{M}} \sum_{\sigma \in \mathcal{E}_{p}} \frac{|\sigma| d_{p \sigma}}{d} d \frac{u_{\sigma}^{n+1}-u_{p}^{n+1}}{d_{p \sigma}} \mathbf{n}_{p \sigma} \cdot \psi_{\sigma}^{n+1}
$$

which gives, thanks to the fact that the terms $u_{\sigma}^{n+1}$ are multiplied by 0 for all $\sigma \in \mathcal{E}_{\text {int }}$ and using $\int_{n \tau}^{(n+1) \tau} \int_{p} \operatorname{div} \psi(x, t) \mathrm{d} x \mathrm{~d} t=\tau \sum_{\sigma \in \mathcal{E}_{p}}|\sigma| \psi_{\sigma}^{n+1} \cdot \mathbf{n}_{p \sigma}$,

$$
\int_{0}^{T} \int_{\mathbb{R}^{d}} G_{m}(x, t) \cdot \psi_{m}(x, t) \mathrm{d} x \mathrm{~d} t=-\int_{0}^{T} \int_{\mathbb{R}^{d}} u_{m}(x, t) \operatorname{div} \psi(x, t) \mathrm{d} x \mathrm{~d} t .
$$


Passing to the limit in the above expression, we get, using weak/strong convergence for the left hand side,

$$
\int_{0}^{T} \int_{\mathbb{R}^{d}} \bar{G}(x, t) \cdot \psi(x, t) \mathrm{d} x \mathrm{~d} t=-\int_{0}^{T} \int_{\mathbb{R}^{d}} \bar{u}(x, t) \operatorname{div} \psi(x, t) \mathrm{d} x \mathrm{~d} t .
$$

This proves that $\nabla \bar{u} \in L^{2}\left(\mathbb{R}^{d} \times(0, T)\right)^{d}$ and that $\nabla \bar{u}=\bar{G}$ for a.e. $(x, t) \in \mathbb{R}^{d} \times(0, T)$. Since $\bar{G}(x, t)=0$ for a.e. $x \notin \Omega \times(0, T)$, we get that $\bar{u} \in L^{2}\left(0, T ; H_{0}^{1}(\Omega)\right)$. Since $\nabla \bar{u} \in L^{2}\left(\mathbb{R}^{d} \times(0, T)\right)^{d}$ is uniquely defined, we get that the whole sequence $G_{m} \rightarrow \nabla \bar{u}$ weakly in $L^{2}\left(\mathbb{R}^{d} \times(0, T)\right)^{d}$, which concludes the proof.

Lemma 4.2 Strong convergence of the approximate gradient norm of regular function interpolation.

Let $\Omega$ be a bounded connected open subset of $\mathbb{R}^{d}$, with $d \in \mathbb{N}^{\star}$ and let $T>0$. Let $(\mathcal{D}, \tau)$ be a space-time discretisation of $\Omega \times(0, T)$ in the sense of Definition 2.2. For any $\varphi \in C_{c}^{\infty}(\Omega \times(0, T))$, we define the discrete interpolation of $\varphi$, denoted $v \in H_{\mathcal{D}, \tau}$, by $v_{p}^{n}=\varphi\left(x_{p}, n \tau\right)$ and $v_{\sigma}^{n}=\varphi\left(x_{\sigma}, n \tau\right)$, and we define $\mathcal{N}_{\mathcal{D}, \tau}$ by

$$
\left.\left.\mathcal{N}_{\mathcal{D}, \tau}(x, t)=N_{p}\left(v^{n+1}\right), \text { for a.e. } x \in p, t \in\right] n \tau,(n+1) \tau\right], \forall p \in \mathcal{M}, \forall n=0, \ldots, N_{T},
$$

Then $\mathcal{N}_{\mathcal{D}, \tau} \rightarrow|\nabla \varphi|$ in $L^{\infty}(\Omega \times(0, T))$ as $h_{\mathcal{D}}$ and $\tau$ tend to 0 .

Proof. We have, for any vector $\mathbf{w} \in \mathbb{R}^{d}$,

$$
|p| \mathbf{w}=\sum_{\sigma \in \mathcal{E}_{p}}|\sigma| \mathbf{w} \cdot\left(x_{\sigma}-x_{p}\right) \mathbf{n}_{p, \sigma} .
$$

Hence we get that

$$
|p||\mathbf{w}|^{2}=\sum_{\sigma \in \mathcal{E}_{p}}|\sigma| \mathbf{w} \cdot\left(x_{\sigma}-x_{p}\right) \mathbf{n}_{p, \sigma} \cdot \mathbf{w}=\sum_{\sigma \in \mathcal{E}_{p}}|\sigma| d_{p \sigma}\left(\mathbf{n}_{p, \sigma} \cdot \mathbf{w}\right)^{2},
$$

thanks to condition (11). This provides that

$$
|p|\left|\nabla \varphi\left(x_{p}, t\right)\right|^{2}=\sum_{\sigma \in \mathcal{E}_{p}}|\sigma| d_{p \sigma}\left(\mathbf{n}_{p, \sigma} \cdot \nabla \varphi\left(x_{p}, t\right)\right)^{2} .
$$

Writing that

$$
\mathbf{n}_{p, \sigma} \cdot \nabla \varphi\left(x_{p}, t\right)=\frac{\varphi\left(x_{\sigma}, t\right)-\varphi\left(x_{p}, t\right)}{d_{p \sigma}}+C_{p}(t) h_{\mathcal{D}}
$$

with $C_{p}(t)$ bounded independently of the discretisation, we conclude the proof of the lemma.

Lemma 4.3 (Strong approximate of the gradient of $\varphi$ ) For all $\varphi \in C_{c}^{\infty}(\Omega \times(0, T)$ ), we denote by $v_{p}^{n}=\varphi\left(x_{p}, n \tau\right)$ and $v_{\sigma}^{n}=\varphi\left(x_{\sigma}, n \tau\right)$. We introduce the approximation

$$
\nabla_{p \sigma}^{n+1} \varphi=\frac{v_{\sigma}^{n+1}-v_{p}^{n+1}}{d_{p \sigma}} \mathbf{n}_{p \sigma}+\nabla \varphi\left(x_{p},(n+1) \tau\right)-\left(\nabla \varphi\left(x_{p},(n+1) \tau\right) \cdot \mathbf{n}_{p \sigma}\right) \mathbf{n}_{p \sigma},
$$

and $\nabla_{\mathcal{D}, \tau} \varphi(x, t)=\nabla_{p \sigma}^{n+1} \varphi$ for $x \in D_{p \sigma}, t \in[n \tau,(n+1) \tau]$.

Then $\nabla_{\mathcal{D}, \tau} \varphi \rightarrow \nabla \varphi$ in $L^{\infty}(\Omega \times(0, T))$ as $h_{\mathcal{D}}$ and $\tau$ tend to 0 .

Proof. The proof relies on (45).

Let us denote by $(\mathrm{HC})$ the following hypotheses:

- Hypotheses (H) are fulfilled.

- The sequence $\left(\mathcal{D}_{m}, \tau_{m}\right)_{m \in \mathbb{N}}$ denotes a sequence of space-time discretisations of $\Omega \times(0, T)$ in the sense of Definition 2.2 such that $h_{\mathcal{D}_{m}}$ and $\tau_{m}>0$ tends to 0 as $m \longrightarrow \infty$. 
- There exists some $\theta>0$ with $\theta<\theta_{\mathcal{D}_{m}}$ for all $m \in \mathbb{N}$, where $\theta_{\mathcal{D}}$ is defined by (12).

- For all $m \in \mathbb{N}$, the family $\left(u_{p}^{n}\right)_{p \in \mathcal{M}, n \in \mathbb{N}}$ is such that (16), (17), (20) and (21), (22), (23) hold and the function $u_{\mathcal{D}_{m}, \tau_{m}}$ is defined by (24).

We can now state the following Lemma, using the compactness properties issued from the above estimates.

Lemma 4.4 (Convergence properties) Let Hypotheses (HC) be fulfilled.

Then there exists a subsequence of $\left(\mathcal{D}_{m}, \tau_{m}\right)_{m \in \mathbb{N}}$, again denoted $\left(\mathcal{D}_{m}, \tau_{m}\right)_{m \in \mathbb{N}}$, there exists a function $\bar{u} \in L^{\infty}\left(0, T ; H_{0}^{1}(\Omega)\right) \cap C^{0}\left(0, T ; L^{2}(\Omega)\right)$, such that $\bar{u}_{t} \in L^{2}(\Omega \times(0, T)), u(., 0)=u_{0}$, and $u_{\mathcal{D}_{m}, \tau_{m}}$ tends to $\bar{u} \in L^{\infty}\left(0, T ; H_{0}^{1}(\Omega)\right)$ in $L^{\infty}\left(0, T ; L^{2}(\Omega)\right)$, and there exists functions $\bar{H} \in L^{2}(\Omega \times(0, T))^{d}, \bar{w} \in L^{2}(\Omega \times$

$(0, T)$ ) such that $H_{\mathcal{D}_{m}, \tau_{m}} \rightarrow \bar{H}$ and $\widetilde{H}_{\mathcal{D}_{m}, \tau_{m}} \rightarrow \bar{H}$ weakly in $L^{2}(\Omega \times(0, T))^{d}$ (see definition (26)), and such that $w_{\mathcal{D}_{m}, \tau_{m}} \rightarrow \bar{w}$ and $z_{\mathcal{D}_{m}, \tau_{m}} \rightarrow \bar{u}_{t}$ weakly in $L^{2}(\Omega \times(0, T))$ as $m \rightarrow \infty$. Moreover, $G_{\mathcal{D}_{m}, \tau_{m}} \rightarrow \nabla \bar{u}$ weakly in $L^{2}(\Omega \times(0, T))^{d}$ (see definition (26)), $N_{\mathcal{D}_{m}, \tau_{m}}-\widetilde{N}_{\mathcal{D}_{m}, \tau_{m}} \rightarrow 0$ in $L^{2}(\Omega \times(0, T)$ ) (see definition (25)) and the following relation holds:

$$
\lim _{m \rightarrow \infty} \int_{0}^{T} \int_{\Omega} \frac{N_{\mathcal{D}_{m}, \tau_{m}}(x, t)^{2}}{f\left(N_{\mathcal{D}_{m}, \tau_{m}}(x, t)\right)} \mathrm{d} x \mathrm{~d} t=\int_{0}^{T} \int_{\Omega} \bar{H}(x, t) \cdot \nabla \bar{u}(x, t) \mathrm{d} x \mathrm{~d} t .
$$

Proof. From the definition of $F$ and Hypotheses (H) (which imply $F(s) \geq s^{2} / 2 b$ ), $u_{\mathcal{D}_{m}, \tau_{m}}(\cdot, t)$ is uniformly bounded in $H_{\mathcal{D}}$ for all $t \in[0, T]$. Hence we can apply Theorem 6.1, which is a generalisation of Ascoli's theorem and shows that the convergence property $u_{\mathcal{D}_{m}, \tau_{m}}(\cdot, t) \rightarrow \bar{u} \in C^{0}\left(0, T ; L^{2}(\Omega)\right)$ holds in $L^{\infty}\left(0, T ; L^{2}(\Omega)\right)$. Thanks to $(16)$, we have $\bar{u}(\cdot, 0)=u_{0}$. We also get, thanks to Lemma 4.1 , that $\bar{u} \in L^{\infty}\left(0, T ; H_{0}^{1}(\Omega)\right)$ and that $G_{\mathcal{D}, \tau} \rightarrow \nabla \bar{u}$ weakly in $L^{2}(\Omega \times(0, T))^{d}$.

From Lemma 3.2 we get that $w_{\mathcal{D}, \tau}$ remains bounded in $L^{2}(\Omega \times(0, T))$ for all $m \in \mathbb{N}$. Therefore there exists a function $\bar{w} \in L^{2}\left((\Omega \times(0, T))\right.$ such that, up to a subsequence of the preceding one, $w_{m} \rightarrow \bar{w}$ weakly in $L^{2}(\Omega \times(0, T))$. Similarly, we have $z_{\mathcal{D}_{m}, \tau_{m}} \rightarrow \bar{u}_{t}$ weakly in $L^{2}(\Omega \times(0, T))$, which shows that $\bar{u}_{t} \in L^{2}(\Omega \times(0, T))$. Similarly, $\widetilde{H}_{\mathcal{D}_{m}, \tau_{m}} \rightarrow \bar{H}$ weakly in $L^{2}(\Omega \times(0, T))^{d}$, up to a subsequence of the preceding one. Note that in the proof below, we drop some indices $m$ for the simplicity of notation.

Let us first focus on the difference between $N_{\mathcal{D}_{m}, \tau_{m}}$ and $\widetilde{N}_{\mathcal{D}_{m}, \tau_{m}}$ on one hand, and that between $\widetilde{H}_{\mathcal{D}_{m}, \tau_{m}}$ and $H_{\mathcal{D}_{m}, \tau_{m}}$ on the other hand. We have, for $x \in p$ and $\left.t \in\right] n \tau,(n+1) \tau[$,

$$
N_{\mathcal{D}, \tau}(x, t)-\widetilde{N}_{\mathcal{D}, \tau}(x, t)=N_{p}\left(u^{n+1}\right)-N_{p}\left(u^{n}\right) .
$$

Using (35), we get the existence of $C>0$ independent of $m$ such that

$$
\left\|N_{\mathcal{D}_{m}, \tau_{m}}-\widetilde{N}_{\mathcal{D}_{m}, \tau_{m}}\right\|_{L^{2}(\Omega \times(0, T))}^{2} \leq C \tau_{m},
$$

which provides

$$
\lim _{m \rightarrow \infty}\left\|N_{\mathcal{D}_{m}, \tau_{m}}-\widetilde{N}_{\mathcal{D}_{m}, \tau_{m}}\right\|_{L^{2}(\Omega \times(0, T))}=0 .
$$

Using the Cauchy-Schwarz inequality, we have

$\int_{0}^{T} \int_{\Omega}\left|\widetilde{H}_{\mathcal{D}_{m}, \tau_{m}}(x, t)-H_{\mathcal{D}_{m}, \tau_{m}}(x, t)\right| \mathrm{d} x \mathrm{~d} t \leq\left\|G_{\mathcal{D}}\right\|_{L^{2}(\Omega \times(0, T))^{d}}\left\|\frac{1}{f\left(\widetilde{N}_{\mathcal{D}_{m}, \tau_{m}}\right)}-\frac{1}{f\left(N_{\mathcal{D}_{m}, \tau_{m}}\right)}\right\|_{L^{2}(\Omega \times(0, T))^{d}}$,

which proves that $\widetilde{H}_{\mathcal{D}_{m}, \tau_{m}}-H_{\mathcal{D}_{m}, \tau_{m}} \rightarrow 0$ in $L^{1}(\Omega \times(0, T))^{d}$ thanks to (48). Note that this shows that $H_{\mathcal{D}_{m}, \tau_{m}} \rightarrow \bar{H}$ weakly in $L^{2}(\Omega \times(0, T))$. One of the difficulties is now to identify $\bar{H}$ with $\nabla u / f(|\nabla u|)$. This will be done in further lemmas, thanks to the property (47) stated in the present lemma, that we have now to prove.

Let $\varphi \in C_{c}^{\infty}(\Omega \times(0, T))$ be given. We denote by $v_{p}^{n}=\varphi\left(x_{p}, n \tau\right)$ and $v_{\sigma}^{n}=\varphi\left(x_{\sigma}, n \tau\right)$. Multiplying (27) by $\tau v_{p}^{n+1}$, summing on $n$ and $p$, we get $T_{1 m}=T_{2 m}$ with

$$
T_{1 m}=\sum_{n=0}^{N_{T}} \tau \sum_{p \in \mathcal{M}} \sum_{\sigma \in \mathcal{E}_{p}} \frac{|\sigma|}{d_{p \sigma}} \frac{u_{\sigma}^{n+1}-u_{p}^{n+1}}{f\left(N_{p}\left(u^{n}\right)\right)}\left(v_{\sigma}^{n+1}-v_{p}^{n+1}\right),
$$


and

$$
T_{2 m}=\sum_{n=0}^{N_{T}} \tau \sum_{p \in \mathcal{M}}|p| w_{p}^{n+1} v_{p}^{n+1} .
$$

Using the approximation $\nabla_{\mathcal{D}, \tau} \varphi$ of $\nabla \varphi$ provided in Lemma 4.3 , we can write that

$$
T_{1 m}=\int_{0}^{T} \int_{\Omega} \widetilde{H}_{\mathcal{D}, \tau} \cdot \nabla_{\mathcal{D}, \tau} \varphi \mathrm{d} x \mathrm{~d} t
$$

Hence, by weak/strong convergence,

$$
\lim _{m \rightarrow \infty} T_{1 m}=\int_{0}^{T} \int_{\Omega} \bar{H} \cdot \nabla \varphi \mathrm{d} x \mathrm{~d} t .
$$

We have on the other hand

$$
\lim _{m \rightarrow \infty} T_{2 m}=\int_{0}^{T} \int_{\Omega} \bar{w} \varphi \mathrm{d} x \mathrm{~d} t
$$

Hence

$$
\int_{0}^{T} \int_{\Omega} \bar{H} \cdot \nabla \varphi \mathrm{d} x \mathrm{~d} t=\int_{0}^{T} \int_{\Omega} \bar{w} \varphi \mathrm{d} x \mathrm{~d} t .
$$

Since the above equality holds for all $\varphi \in C_{c}^{\infty}(\Omega \times(0, T))$, it also holds by density for all $v \in L^{2}\left(0, T ; H_{0}^{1}(\Omega)\right)$. Hence we get

$$
\int_{0}^{T} \int_{\Omega} \bar{H} \cdot \nabla \bar{u} \mathrm{~d} x \mathrm{~d} t=\int_{0}^{T} \int_{\Omega} \bar{w} \bar{u} \mathrm{~d} x \mathrm{~d} t
$$

We now multiply $(27)$ by $\tau u_{p}^{n+1}$, sum on $n$ and $p$. We get $\widetilde{T}_{3 m}=T_{4 m}$ with $\widetilde{T}_{3 m}$ defined by

$$
\widetilde{T}_{3 m}=\sum_{n=0}^{N_{T}} \tau \sum_{p \in \mathcal{M}} \sum_{\sigma \in \mathcal{E}_{p}} \frac{|\sigma|}{d_{p \sigma}} \frac{\left(u_{\sigma}^{n+1}-u_{p}^{n+1}\right)^{2}}{f\left(N_{p}\left(u^{n}\right)\right)}=\int_{0}^{T} \int_{\Omega} \frac{N_{\mathcal{D}, \tau}(x, t)^{2}}{f\left(\widetilde{N}_{\mathcal{D}, \tau}(x, t)\right)} \mathrm{d} x \mathrm{~d} t,
$$

and

$$
T_{4 m}=\sum_{n=0}^{N_{T}} \tau \sum_{p \in \mathcal{M}}|p| w_{p}^{n+1} u_{p}^{n+1} .
$$

We have, by weak/strong convergence,

$$
\lim _{m \rightarrow \infty} T_{4 m}=\int_{0}^{T} \int_{\Omega} \bar{w} \bar{u} \mathrm{~d} x \mathrm{~d} t
$$

which leads, using (49), to

$$
\lim _{m \rightarrow \infty} T_{3 m}=\int_{0}^{T} \int_{\Omega} \bar{w} \bar{u} \mathrm{~d} x \mathrm{~d} t=\int_{0}^{T} \int_{\Omega} \bar{H} \cdot \nabla \bar{u} \mathrm{~d} x \mathrm{~d} t,
$$

We now define

$$
T_{3 m}=\int_{0}^{T} \int_{\Omega} \frac{N_{\mathcal{D}_{m}, \tau_{m}}(x, t)^{2}}{f\left(N_{\mathcal{D}_{m}, \tau_{m}}(x, t)\right)} \mathrm{d} x \mathrm{~d} t=\sum_{n=0}^{N_{T}} \tau \sum_{p \in \mathcal{M}} \sum_{\sigma \in \mathcal{E}_{p}} \frac{|\sigma|}{d_{p \sigma}} \frac{\left(u_{\sigma}^{n+1}-u_{p}^{n+1}\right)^{2}}{f\left(N_{p}\left(u^{n+1}\right)\right)}
$$

again dropping some indices $m$ for the simplicity of notation. Let us now prove that $\widetilde{T}_{3 m}$ and $T_{3 m}$ have the same limit. Writing

$$
\widetilde{T}_{3 m}-T_{3 m}=-\tau \sum_{p \in \mathcal{M}}|p| \frac{N_{p}\left(u^{N_{T}+1}\right)^{2}}{f\left(N_{p}\left(u^{N_{T}+1}\right)\right)}+\sum_{n=0}^{N_{T}} \tau \sum_{p \in \mathcal{M}}|p| \frac{N_{p}\left(u^{n+1}\right)^{2}-N_{p}\left(u^{n}\right)^{2}}{f\left(N_{p}\left(u^{n}\right)\right)}+\tau \sum_{p \in \mathcal{M}}|p| \frac{N_{p}\left(u^{0}\right)^{2}}{f\left(N_{p}\left(u^{0}\right)\right)}
$$


we get, using (35) for the study of the first term in the right hand side of the above equation, (48) for the study of the second term and (38) for the third one, that

$$
\lim _{m \rightarrow \infty}\left(\widetilde{T}_{3 m}-T_{3 m}\right)=0,
$$

Hence we also get that

$$
\lim _{m \rightarrow \infty} T_{3 m}=\int_{0}^{T} \int_{\Omega} \bar{w} \bar{u} \mathrm{~d} x \mathrm{~d} t=\int_{0}^{T} \int_{\Omega} \bar{H} \cdot \nabla \bar{u} \mathrm{~d} x \mathrm{~d} t,
$$

which completes the proof of (47).

The problem is now to show the strong convergence in $L^{2}(\Omega \times(0, T))$ of $N_{\mathcal{D}}\left(u_{\mathcal{D}, \tau}\right)$ to $|\nabla \bar{u}|$. This will result from property (47), from Minty trick and from Leray-Lions trick. Let us start with the following property:

Lemma 4.5 For all $u, v \in H_{\mathcal{D}}$,

$$
\left.\sum_{p \in \mathcal{M}} \sum_{\sigma \in \mathcal{E}_{p}} \frac{|\sigma|}{d_{p \sigma}}\left(\frac{u_{\sigma}-u_{p}}{f\left(N_{p}(u)\right)}-\frac{v_{\sigma}-v_{p}}{f\left(N_{p}(v)\right)}\right)\right)\left(u_{\sigma}-u_{p}-v_{\sigma}+v_{p}\right) \geq 0 .
$$

Proof. We have that

$$
\begin{aligned}
& \left.\sum_{\sigma \in \mathcal{E}_{p}} \frac{|\sigma|}{d_{p \sigma}}\left(\frac{u_{\sigma}-u_{p}}{f\left(N_{p}(u)\right)}-\frac{v_{\sigma}-v_{p}}{f\left(N_{p}(v)\right)}\right)\right)\left(u_{\sigma}-u_{p}-v_{\sigma}+v_{p}\right) \\
& =\sum_{\sigma \in \mathcal{E}_{p}}\left(\frac{|\sigma|}{d_{p \sigma}} \frac{\left(u_{\sigma}-u_{p}\right)^{2}}{f\left(N_{p}(u)\right)}+\frac{|\sigma|}{d_{p \sigma}} \frac{\left(v_{\sigma}-v_{p}\right)^{2}}{f\left(N_{p}(v)\right)}-\frac{|\sigma|}{d_{p \sigma}} \frac{\left(u_{\sigma}-u_{p}\right)\left(v_{\sigma}-v_{p}\right)}{f\left(N_{p}(u)\right)}-\frac{|\sigma|}{d_{p \sigma}} \frac{\left(u_{\sigma}-u_{p}\right)\left(v_{\sigma}-v_{p}\right)}{f\left(N_{p}(v)\right)}\right) .
\end{aligned}
$$

Applying the Cauchy-Schwarz inequality, we get

$$
\begin{aligned}
\left.\sum_{\sigma \in \mathcal{E}_{p}} \frac{|\sigma|}{d_{p \sigma}}\left(\frac{u_{\sigma}-u_{p}}{f\left(N_{p}(u)\right)}-\frac{v_{\sigma}-v_{p}}{f\left(N_{p}(v)\right)}\right)\right)\left(u_{\sigma}-u_{p}-v_{\sigma}+v_{p}\right) \geq & \frac{|p| N_{p}(u)^{2}}{f\left(N_{p}(u)\right)}+\frac{|p| N_{p}(v)^{2}}{f\left(N_{p}(v)\right)} \\
& -\frac{|p| N_{p}(u) N_{p}(v)}{f\left(N_{p}(u)\right)}-\frac{|p| N_{p}(u) N_{p}(v)}{f\left(N_{p}(v)\right)}
\end{aligned}
$$

which gives

$$
\begin{aligned}
& \left.\sum_{\sigma \in \mathcal{E}_{p}} \frac{|\sigma|}{d_{p \sigma}}\left(\frac{u_{\sigma}-u_{p}}{f\left(N_{p}(u)\right)}-\frac{v_{\sigma}-v_{p}}{f\left(N_{p}(v)\right)}\right)\right)\left(u_{\sigma}-u_{p}-v_{\sigma}+v_{p}\right) \geq \\
& |p|\left(\frac{N_{p}(u)}{f\left(N_{p}(u)\right)}-\frac{N_{p}(v)}{f\left(N_{p}(v)\right)}\right)\left(N_{p}(u)-N_{p}(v)\right) .
\end{aligned}
$$

This last expression is non negative thanks to the hypotheses $(\mathrm{H})$.

We now continue with the use of Minty trick.

Lemma 4.6 Let Hypotheses $(H C)$ be fulfilled. We assume that the sequence $\left(\mathcal{D}_{m}, \tau_{m}\right)_{m \in \mathbb{N}}$ denotes an extracted sub-sequence, the existence of which is provided by Lemma 4.4. Let $\varphi \in C_{c}^{\infty}(\Omega \times(0, T))$ be given. We denote by $v_{p}^{n}=\varphi\left(x_{p}, n \tau\right)$ and $v_{\sigma}^{n}=\varphi\left(x_{\sigma}, n \tau\right)$ and by

$$
\left.T_{m}=\sum_{n=0}^{N_{T}} \tau \sum_{p \in \mathcal{M}} \sum_{\sigma \in \mathcal{E}_{p}} \frac{|\sigma|}{d_{p \sigma}}\left(\frac{u_{\sigma}^{n+1}-u_{p}^{n+1}}{f\left(N_{p}\left(u^{n+1}\right)\right)}-\frac{v_{\sigma}^{n+1}-v_{p}^{n+1}}{f\left(N_{p}\left(v^{n+1}\right)\right)}\right)\right)\left(u_{\sigma}^{n+1}-u_{p}^{n+1}-v_{\sigma}^{n+1}+v_{p}^{n+1}\right) .
$$

Then the following holds

$$
\lim _{m \rightarrow \infty} T_{m}=\int_{0}^{T} \int_{\Omega}\left(\bar{H}-\frac{\nabla \varphi}{f(|\nabla \varphi|)}\right)(\nabla \bar{u}-\nabla \varphi) \mathrm{d} x \mathrm{~d} t,
$$


and

$$
\int_{0}^{T} \int_{\Omega} \bar{H} \cdot \nabla v \mathrm{~d} x \mathrm{~d} t=\int_{0}^{T} \int_{\Omega} \frac{\nabla \bar{u}}{f(|\nabla \bar{u}|)} \cdot \nabla v \mathrm{~d} x \mathrm{~d} t, \forall v \in L^{2}\left(0, T ; H_{0}^{1}(\Omega)\right) .
$$

Proof. We remark that $T_{m}=T_{3 m}-T_{5 m}-T_{6 m}+T_{7 m}$, with $T_{3 m}$ defined by (50) and

$$
\begin{aligned}
& T_{5 m}=\sum_{n=0}^{N_{T}} \tau \sum_{p \in \mathcal{M}} \sum_{\sigma \in \mathcal{E}_{p}} \frac{|\sigma|}{d_{p \sigma}}\left(\frac{u_{\sigma}^{n+1}-u_{p}^{n+1}}{f\left(N_{p}\left(u^{n+1}\right)\right)}\right)\left(v_{\sigma}^{n+1}-v_{p}^{n+1}\right), \\
& T_{6 m}=\sum_{n=0}^{N_{T}} \tau \sum_{p \in \mathcal{M}} \sum_{\sigma \in \mathcal{E}_{p}} \frac{|\sigma|}{d_{p \sigma}}\left(\frac{v_{\sigma}^{n+1}-v_{p}^{n+1}}{f\left(N_{p}\left(v^{n+1}\right)\right)}\right)\left(u_{\sigma}^{n+1}-u_{p}^{n+1}\right), \\
& T_{7 m}=\sum_{n=0}^{N_{T}} \tau \sum_{p \in \mathcal{M}} \sum_{\sigma \in \mathcal{E}_{p}} \frac{|\sigma|}{d_{p \sigma}}\left(\frac{v_{\sigma}^{n+1}-v_{p}^{n+1}}{f\left(N_{p}\left(v^{n+1}\right)\right)}\right)\left(v_{\sigma}^{n+1}-v_{p}^{n+1}\right) .
\end{aligned}
$$

We have that $N_{\mathcal{D}}\left(v_{\mathcal{D}, \tau}\right) \rightarrow|\nabla \varphi|$ in $L^{\infty}(\Omega \times(0, T))$, which leads to

$$
\begin{gathered}
\lim _{m \rightarrow \infty} T_{5 m}=\int_{0}^{T} \int_{\Omega} \bar{H} \cdot \nabla \varphi \mathrm{d} x \mathrm{~d} t \\
\lim _{m \rightarrow \infty} T_{6 m}=\int_{0}^{T} \int_{\Omega} \frac{\nabla \varphi}{f(|\nabla \varphi|)} \cdot \nabla \bar{u} \mathrm{~d} x \mathrm{~d} t, \\
\lim _{m \rightarrow \infty} T_{7 m}=\int_{0}^{T} \int_{\Omega} \frac{\nabla \varphi}{f(|\nabla \varphi|)} \cdot \nabla \varphi \mathrm{d} x \mathrm{~d} t .
\end{gathered}
$$

Hence, gathering the above results, we get (52). We have that $T_{m} \geq 0$ thanks to Lemma 4.5. Hence (52) provides

$$
\int_{0}^{T} \int_{\Omega}\left(\bar{H}-\frac{\nabla \varphi}{f(|\nabla \varphi|)}\right)(\nabla \bar{u}-\nabla \varphi) \mathrm{d} x \mathrm{~d} t \geq 0
$$

and therefore we get by density

$$
\forall v \in L^{2}\left(0, T ; H_{0}^{1}(\Omega)\right), \int_{0}^{T} \int_{\Omega}\left(\bar{H}-\frac{\nabla v}{f(|\nabla v|)}\right)(\nabla \bar{u}-\nabla v) \mathrm{d} x \mathrm{~d} t \geq 0 .
$$

We can now apply Minty's trick, taking in (54) $v=\bar{u}-\lambda \psi$, with $\lambda>0$ and $\psi \in C_{c}^{\infty}(\Omega \times(0, T))$. We get, dividing by $\lambda$,

$$
\int_{0}^{T} \int_{\Omega}\left(\bar{H}-\frac{\nabla(\bar{u}-\lambda \psi)}{f(|\nabla(\bar{u}-\lambda \psi)|)}\right) \nabla \psi \mathrm{d} x \mathrm{~d} t \geq 0 .
$$

We can let $\lambda \longrightarrow 0$ in the above inequality, using Lebesgue's dominated convergence theorem. We then get

$$
\int_{0}^{T} \int_{\Omega}\left(\bar{H}-\frac{\nabla \bar{u}}{f(|\nabla \bar{u}|)}\right) \nabla \psi \mathrm{d} x \mathrm{~d} t \geq 0 .
$$

Since this also holds for $-\psi$, we get

$$
\int_{0}^{T} \int_{\Omega}\left(\bar{H}-\frac{\nabla \bar{u}}{f(|\nabla \bar{u}|)}\right) \nabla \psi \mathrm{d} x \mathrm{~d} t=0 .
$$

The above equality can again be extended to all $v \in L^{2}\left(0, T ; H_{0}^{1}(\Omega)\right)$, which achieves the proof of (53). We have now the following lemma, which uses Leray-Lions trick. 
Lemma 4.7 Under the same hypotheses as Lemma 4.6, $N_{\mathcal{D}_{m}, \tau_{m}} \rightarrow|\nabla \bar{u}|$ in $L^{2}(\Omega \times(0, T))$ as $m$ tends to $\infty$.

Proof. For a given $m \in \mathbb{N}$, we drop the indices $m$ in $\mathcal{D}, \tau$ in order to lighten the notation. Let $\varphi \in C_{c}^{\infty}(\Omega \times(0, T))$, we denote by $v_{p}^{n}=\varphi\left(x_{p}, n \tau\right)$ and $v_{\sigma}^{n}=\varphi\left(x_{\sigma}, n \tau\right)$. Let us denote

$$
T_{8 m}=\sum_{n=0}^{N_{T}} \tau \sum_{p \in \mathcal{M}}|p|\left(\frac{N_{p}\left(u^{n+1}\right)}{f\left(N_{p}\left(u^{n+1}\right)\right)}-\frac{N_{p}\left(v^{n+1}\right)}{f\left(N_{p}\left(v^{n+1}\right)\right)}\right)\left(N_{p}\left(u^{n+1}\right)-N_{p}\left(v^{n+1}\right)\right) .
$$

We have

$$
T_{8 m}=\int_{0}^{T} \int_{\Omega}\left(\frac{N_{\mathcal{D}}\left(u_{\mathcal{D}, \tau}\right)}{f\left(N_{\mathcal{D}}\left(u_{\mathcal{D}, \tau}\right)\right)}-\frac{N_{\mathcal{D}}\left(v_{\mathcal{D}, \tau}\right)}{f\left(N_{\mathcal{D}}\left(v_{\mathcal{D}, \tau}\right)\right)}\right)\left(N_{\mathcal{D}}\left(u_{\mathcal{D}, \tau}\right)-N_{\mathcal{D}}\left(v_{\mathcal{D}, \tau}\right)\right) \mathrm{d} x \mathrm{~d} t .
$$

From the proof of lemma 4.5, we have

$$
0 \leq T_{8 m} \leq T_{m} .
$$

We write $T_{8 m}=T_{9 m}-T_{10 m}-T_{11 m}$, with

$$
\begin{aligned}
& T_{9 m}=\int_{0}^{T} \int_{\Omega}\left(\frac{N_{\mathcal{D}}\left(u_{\mathcal{D}, \tau}\right)}{f\left(N_{\mathcal{D}}\left(u_{\mathcal{D}, \tau}\right)\right)}-\frac{|\nabla \bar{u}|}{f(|\nabla \bar{u}|)}\right)\left(N_{\mathcal{D}}\left(u_{\mathcal{D}, \tau}\right)-|\nabla \bar{u}|\right) \mathrm{d} x \mathrm{~d} t, \\
& T_{10 m}=-\int_{0}^{T} \int_{\Omega}\left(\frac{|\nabla \varphi|^{2}}{f(|\nabla \varphi|)}-\frac{|\nabla \bar{u}|^{2}}{f(|\nabla \bar{u}|)}\right) \mathrm{d} x \mathrm{~d} t \\
& +\int_{0}^{T} \int_{\Omega} \frac{N_{\mathcal{D}}\left(u_{\mathcal{D}, \tau}\right)}{f\left(N_{\mathcal{D}}\left(u_{\mathcal{D}, \tau}\right)\right)}(|\nabla \varphi|-|\nabla \bar{u}|) \mathrm{d} x \mathrm{~d} t \\
& +\int_{0}^{T} \int_{\Omega} N_{\mathcal{D}}\left(u_{\mathcal{D}, \tau}\right)\left(\frac{|\nabla \varphi|}{f(|\nabla \varphi|)}-\frac{|\nabla \bar{u}|}{f(|\nabla \bar{u}|)}\right) \mathrm{d} x \mathrm{~d} t, \\
& T_{11 m}=\int_{0}^{T} \int_{\Omega}\left(\frac{|\nabla \varphi|^{2}}{f(|\nabla \varphi|)}-\frac{N_{\mathcal{D}}\left(v_{\mathcal{D}, \tau}\right)^{2}}{f\left(N_{\mathcal{D}}\left(v_{\mathcal{D}, \tau}\right)\right)}\right) \mathrm{d} x \mathrm{~d} t \\
& -\int_{0}^{T} \int_{\Omega} \frac{N_{\mathcal{D}}\left(u_{\mathcal{D}, \tau}\right)}{f\left(N_{\mathcal{D}}\left(u_{\mathcal{D}, \tau}\right)\right)}\left(|\nabla \varphi|-N_{\mathcal{D}}\left(v_{\mathcal{D}, \tau}\right)\right) \mathrm{d} x \mathrm{~d} t \\
& -\int_{0}^{T} \int_{\Omega} N_{\mathcal{D}}\left(u_{\mathcal{D}, \tau}\right)\left(\frac{|\nabla \varphi|}{f(|\nabla \varphi|)}-\frac{N_{\mathcal{D}}\left(v_{\mathcal{D}, \tau}\right)}{f\left(N_{\mathcal{D}}\left(v_{\mathcal{D}, \tau}\right)\right)}\right) \mathrm{d} x \mathrm{~d} t,
\end{aligned}
$$

We then deduce, using Cauchy-Schwarz inequalities and estimates on the scheme,

$$
0 \leq T_{9 m} \leq T_{m}+C|| \nabla \varphi|-| \nabla \bar{u}\left|\left\|_{L^{2}(\Omega \times(0, T))}+C|||\nabla \varphi|-N_{\mathcal{D}}\left(v_{\mathcal{D}, \tau}\right)\right\|_{L^{2}(\Omega \times(0, T))} .\right.
$$

Hence, passing to the limit $m \rightarrow \infty$, since $N_{\mathcal{D}}\left(v_{\mathcal{D}, \tau}\right) \rightarrow|\nabla \varphi|$ in $L^{\infty}(\Omega \times(0, T))$, we get

$$
0 \leq \limsup _{m \rightarrow \infty} T_{9 m} \leq \int_{0}^{T} \int_{\Omega}\left(\frac{\nabla \bar{u}}{f(|\nabla \bar{u}|)}-\frac{\nabla \varphi}{f(|\nabla \varphi|)}\right)(\nabla \bar{u}-\nabla \varphi) \mathrm{d} x \mathrm{~d} t+C|| \nabla \varphi|-| \nabla \bar{u} \mid \|_{L^{2}(\Omega \times(0, T))} .
$$

Since this holds for any $\varphi \in C_{c}^{\infty}(\Omega \times(0, T))$, we can let $\varphi \rightarrow \bar{u}$ in $L^{2}\left(0, T ; H_{0}^{1}(\Omega)\right)$. Then the right hand side of the above inequality tends to 0 , and we get

$$
\lim _{m \rightarrow \infty} \int_{0}^{T} \int_{\Omega}\left(\frac{N_{\mathcal{D}_{m}}\left(u_{\mathcal{D}_{m}, \tau_{m}}\right)}{f\left(N_{\mathcal{D}_{m}}\left(u_{\mathcal{D}_{m}, \tau_{m}}\right)\right)}-\frac{|\nabla \bar{u}|}{f(|\nabla \bar{u}|)}\right)\left(N_{\mathcal{D}_{m}}\left(u_{\mathcal{D}_{m}, \tau_{m}}\right)-|\nabla \bar{u}|\right) \mathrm{d} x \mathrm{~d} t=0 .
$$

Simple conclusion of the proof in the case where $\left(\frac{c}{f(c)}-\frac{d}{f(d)}\right)(c-d) \geq \alpha(c-d)^{2}$ (this holds if the function $x \mapsto x / f(x)$ has its derivative greater or equal to $\alpha>0$; this is satisfied by the example provided in (6)). We immediately get the conclusion of the lemma. 
More complex conclusion of the proof in the general case. Let us now apply lemma (6.1). We get that $N_{\mathcal{D}_{m}}\left(u_{\mathcal{D}_{m}, \tau_{m}}\right)$ converges a.e. to $|\nabla \bar{u}|$. We then remark that, thanks to (47) and (53), we have

$$
\lim _{m \rightarrow \infty} \int_{0}^{T} \int_{\Omega} \frac{N_{\mathcal{D}_{m}}\left(u_{\mathcal{D}_{m}, \tau_{m}}\right)^{2}}{f\left(N_{\mathcal{D}_{m}}\left(u_{\mathcal{D}_{m}, \tau_{m}}\right)\right)} \mathrm{d} x \mathrm{~d} t=\int_{0}^{T} \int_{\Omega} \frac{|\nabla \bar{u}|^{2}}{f(|\nabla \bar{u}|)} \mathrm{d} x \mathrm{~d} t .
$$

We now apply lemma 6.2, which shows that $\frac{N_{\mathcal{D}_{m}}\left(u_{\mathcal{D}_{m}, \tau_{m}}\right)^{2}}{f\left(N_{\mathcal{D}_{m}}\left(u_{\mathcal{D}_{m}, \tau_{m}}\right)\right)} \rightarrow \frac{|\nabla \bar{u}|^{2}}{f(|\nabla \bar{u}|)}$ in $L^{1}(\Omega \times(0, T))$. This $L^{1}$ convergence gives the equi-integrability of the family of functions $\frac{N_{\mathcal{D}_{m}}\left(u_{\mathcal{D}_{m}, \tau_{m}}\right)^{2}}{f\left(N_{\mathcal{D}_{m}}\left(u_{\mathcal{D}_{m}, \tau_{m}}\right)\right)}$, which, in turn, gives that the family of functions $N_{\mathcal{D}_{m}}\left(u_{\mathcal{D}_{m}, \tau_{m}}\right)^{2}$ is equi-integrable. Finally, we obtain (using Vitali's theorem) the convergence of $N_{\mathcal{D}_{m}}\left(u_{\mathcal{D}_{m}, \tau_{m}}\right)$ to $|\nabla \bar{u}|$ in $L^{2}(\Omega)$, as $m \rightarrow \infty$. This completes the proof.

We can now conclude the convergence of the scheme. We introduce the following strongly convergent approximation for the gradient of the unknown:

$$
\begin{aligned}
& \widehat{G}_{\mathcal{D}, \tau}(x, t)=\frac{1}{|p|} \sum_{\sigma \in \mathcal{E}_{p}}|\sigma|\left(u_{\sigma}^{n+1}-u_{p}^{n+1}\right) \mathbf{n}_{p \sigma}, \\
& \text { for a.e. } x \in p, \text { for a.e. } t \in] n \tau,(n+1) \tau[, \forall p \in \mathcal{M}, \forall n \in \mathbb{N} .
\end{aligned}
$$

(recall that $G_{\mathcal{D}, \tau}(x, t)$ is only weakly convergent).

Theorem 4.1 Let Hypotheses $(\mathrm{HC})$ be fulfilled. We assume that the sequence $\left(\mathcal{D}_{m}, \tau_{m}\right)_{m \in \mathbb{N}}$ denotes an extracted sub-sequence, the existence of which is provided by Lemma 4.4.

Then the function $\bar{u} \in L^{\infty}\left(0, T ; H_{0}^{1}(\Omega)\right)$, such that $u_{\mathcal{D}_{m}, \tau_{m}} \rightarrow \bar{u}$ in $L^{\infty}\left(0, T ; L^{2}(\Omega)\right)$, is a weak solution of (1)-(2)-(3) in the sense of Definition 1.1. Moreover, $\widehat{G}_{\mathcal{D}_{m}, \tau_{m}} \rightarrow \nabla \bar{u}$ in $L^{2}(\Omega \times(0, T))^{d}$ (see (55)) and $N_{\mathcal{D}_{m}, \tau_{m}} \rightarrow|\nabla \bar{u}|, \tilde{N}_{\mathcal{D}_{m}, \tau_{m}} \rightarrow|\nabla \bar{u}|$ in $L^{2}(\Omega \times(0, T))$.

ProOF.

Using Lemma (4.6), we get that

$$
\int_{0}^{T} \int_{\Omega} \frac{\nabla \bar{u}}{f(|\nabla \bar{u}|)} \cdot \nabla v \mathrm{~d} x \mathrm{~d} t=\int_{0}^{T} \int_{\Omega} \bar{w} v \mathrm{~d} x \mathrm{~d} t, \forall v \in L^{2}\left(0, T ; H_{0}^{1}(\Omega)\right),
$$

Thanks to Lemma 4.7, we get that $\bar{w}=\left(r-u_{t}\right) / g(|\nabla u|)$, and the proof that $\bar{u}$ is a weak solution of (1)-(2)-(3) in the sense of Definition 1.1 is complete.

Let us turn to the proof of the strong convergence of $\widehat{G}_{\mathcal{D}_{m}, \tau_{m}}$. Let us first remark that, thanks to (44), the expression of $\widehat{G}_{\mathcal{D}, \tau}$, applied to the interpolation of some regular function $\varphi$, is strongly consistent with $\nabla \varphi$.

We can then follow the reasoning of [20] in order to prove the strong convergence of $\widehat{G}_{\mathcal{D}_{m}, \tau_{m}}$ to $\nabla \bar{u}$. Indeed, let $\varphi \in C_{c}^{\infty}(\Omega \times(0, T))$ be given (this function is devoted to approximate $\bar{u}$ in $L^{2}\left(0, T ; H_{0}^{1}(\Omega)\right)$ ). We define, for $m \in \mathbb{N}, p \in \mathcal{M}_{m}$ and $\sigma \in \mathcal{E}_{m}$, the values $v_{p}^{n}=\varphi\left(x_{p}, n \tau\right)$ and $v_{\sigma}^{n}=\varphi\left(x_{\sigma}, n \tau\right)$, which are used in the definition of

$$
\begin{aligned}
& \widehat{\nabla}_{\mathcal{D}_{m}, \tau_{m}} \varphi(x, t)=\frac{1}{|p|} \sum_{\sigma \in \mathcal{E}_{p}}|\sigma|\left(v_{\sigma}^{n+1}-v_{p}^{n+1}\right) \mathbf{n}_{p \sigma}, \\
& \text { for a.e. } x \in p, \text { for a.e. } t \in] n \tau,(n+1) \tau[, \forall p \in \mathcal{M}, \forall n \in \mathbb{N} .
\end{aligned}
$$

Thanks to the Cauchy-Schwarz inequality, we have

$$
\int_{0}^{T} \int_{\Omega}\left|\widehat{G}_{\mathcal{D}_{m}, \tau_{m}}(x, t)-\nabla \bar{u}(x, t)\right|^{2} \mathrm{~d} x \mathrm{~d} t \leq 3\left(T_{12 m}+T_{13 m}+T_{14 m}\right),
$$


with $T_{12 m}=\int_{0}^{T} \int_{\Omega}\left|\widehat{G}_{\mathcal{D}_{m}, \tau_{m}}(x, t)-\widehat{\nabla}_{\mathcal{D}_{m}, \tau_{m}} \varphi(x, t)\right|^{2} \mathrm{~d} x \mathrm{~d} t, T_{13 m}=\int_{0}^{T} \int_{\Omega}\left|\widehat{\nabla}_{\mathcal{D}_{m}, \tau_{m}} \varphi(x, t)-\nabla \varphi(x, t)\right|^{2} \mathrm{~d} x \mathrm{~d} t$, and $T_{14 m}=\int_{0}^{T} \int_{\Omega}|\nabla \varphi(x, t)-\nabla \bar{u}(x, t)|^{2} \mathrm{~d} x \mathrm{~d} t$. In a similar way as in Lemma 4.2, we have $\lim _{m \rightarrow \infty} T_{13 m}=$ 0 . Using the Cauchy-Schwarz inequality, we have

$$
T_{12 m} \leq d\left\langle u_{\mathcal{D}_{m}, \tau_{m}}-v_{\mathcal{D}_{m}, \tau_{m}}, u_{\mathcal{D}_{m}, \tau_{m}}-v_{\mathcal{D}_{m}, \tau_{m}}\right\rangle,
$$

defining $\langle\cdot, \cdot\rangle$ by

$$
\langle u, v\rangle=\sum_{n=0}^{N_{T}} \tau \sum_{p \in \mathcal{M}} \sum_{\sigma \in \mathcal{E}_{p}} \frac{|\sigma|}{d_{p \sigma}}\left(u_{\sigma}^{n+1}-u_{p}^{n+1}\right)\left(v_{\sigma}^{n+1}-v_{p}^{n+1}\right) .
$$

Developing equation (58), we obtain

$$
T_{12 m} \leq d\left(\left\langle u_{\mathcal{D}_{m}, \tau_{m}}, u_{\mathcal{D}_{m}, \tau_{m}}\right\rangle-2\left\langle u_{\mathcal{D}_{m}, \tau_{m}}, v_{\mathcal{D}_{m}, \tau_{m}}\right\rangle+\left\langle v_{\mathcal{D}_{m}, \tau_{m}}, v_{\mathcal{D}_{m}, \tau_{m}}\right\rangle\right) .
$$

Since, from the definitions (26) and (46), we have

$$
\left\langle u_{\mathcal{D}_{m}, \tau_{m}}, v_{\mathcal{D}_{m}, \tau_{m}}\right\rangle=\int_{0}^{T} \int_{\Omega} G_{\mathcal{D}_{m}, \tau_{m}}(x, t) \cdot \nabla_{\mathcal{D}_{m}, \tau_{m}} \varphi(x, t) \mathrm{d} x \mathrm{~d} t,
$$

using the convergence properties of $G_{\mathcal{D}_{m}, \tau_{m}}$ and $\nabla_{\mathcal{D}_{m}, \tau_{m}} \varphi$, we obtain

$$
\lim _{m \rightarrow \infty}\left\langle u_{\mathcal{D}_{m}, \tau_{m}}, v_{\mathcal{D}_{m}, \tau_{m}}\right\rangle=\int_{0}^{T} \int_{\Omega} \nabla \bar{u}(x, t) \cdot \nabla \varphi(x, t) \mathrm{d} x \mathrm{~d} t .
$$

Since $\left\langle v_{\mathcal{D}, \tau}, v_{\mathcal{D}, \tau}\right\rangle=\int_{0}^{T} \int_{\Omega} \mathcal{N}_{\mathcal{D}, \tau}(x, t)^{2} \mathrm{~d} x \mathrm{~d} t$, Lemma 4.2 states that

$$
\lim _{m \rightarrow \infty}\left\langle v_{\mathcal{D}_{m}, \tau_{m}}, v_{\mathcal{D}_{m}, \tau_{m}}\right\rangle=\int_{0}^{T} \int_{\Omega}|\nabla \varphi(x, t)|^{2} \mathrm{~d} x \mathrm{~d} t .
$$

Since $\left\langle u_{\mathcal{D}, \tau}, u_{\mathcal{D}, \tau}\right\rangle=\int_{0}^{T} \int_{\Omega} N_{\mathcal{D}, \tau}(x, t)^{2} \mathrm{~d} x \mathrm{~d} t$, we get from Lemma 4.7 that

$$
\lim _{m \rightarrow \infty}\left\langle u_{\mathcal{D}_{m}, \tau_{m}}, u_{\mathcal{D}_{m}, \tau_{m}}\right\rangle=\int_{0}^{T} \int_{\Omega}|\nabla \bar{u}(x, t)|^{2} \mathrm{~d} x \mathrm{~d} t .
$$

Gathering the above results, we get that

$$
\lim _{m \rightarrow \infty}\left\langle u_{\mathcal{D}_{m}, \tau_{m}}-v_{\mathcal{D}_{m}, \tau_{m}}, u_{\mathcal{D}_{m}, \tau_{m}}-v_{\mathcal{D}_{m}, \tau_{m}}\right\rangle=\int_{0}^{T} \int_{\Omega}|\nabla \bar{u}-\nabla \varphi|^{2} \mathrm{~d} x \mathrm{~d} t
$$

which yields

$$
\limsup _{m \rightarrow \infty} T_{12 m} \leq d \int_{0}^{T} \int_{\Omega}|\nabla \bar{u}-\nabla \varphi|^{2} \mathrm{~d} x \mathrm{~d} t .
$$

From the above results, we obtain that

$$
\int_{0}^{T} \int_{\Omega}\left|\widehat{G}_{\mathcal{D}_{m}, \tau_{m}}(x, t)-\nabla \bar{u}(x, t)\right|^{2} \mathrm{~d} x \mathrm{~d} t \leq 3(d+1) \int_{0}^{T} \int_{\Omega}|\nabla \varphi(x, t)-\nabla \bar{u}(x, t)|^{2} \mathrm{~d} x \mathrm{~d} t+T_{15 m},
$$

with (noting that $\varphi$ is fixed) $\lim _{m \rightarrow \infty} T_{15 m}=0$. Let $\varepsilon>0$; we may choose $\varphi$ such that $\int_{0}^{T} \int_{\Omega} \mid \nabla \varphi(x, t)-$ $\left.\nabla \bar{u}(x, t)\right|^{2} \mathrm{~d} x \mathrm{~d} t \leq \varepsilon$, and we may then choose $m$ large enough so that $T_{15 m} \leq \varepsilon$. This completes the proof that

$$
\lim _{m \rightarrow \infty} \int_{0}^{T} \int_{\Omega}\left|\widehat{G}_{\mathcal{D}_{m}, \tau_{m}}(x, t)-\nabla \bar{u}(x, t)\right|^{2} \mathrm{~d} x \mathrm{~d} t=0 .
$$

Remark 4.1 The above convergence theorem also holds for the fully implicit scheme, under almost the same hypotheses (the hypothesis that $f$ is non-decreasing is not necessary). 


\section{$5 \quad$ Numerical experiments}

In this section we present two numerical examples to illustrate the properties of the proposed finite volume schemes. In both examples we use the semi-implicit and fully implicit scheme and compute the errors and experimental order of convergence (EOC) in several functional spaces. In the tables below $n$ is number of finite volumes along each boundary side which means that $n^{2}$ is a total number of finite volumes, because we consider square domain $\Omega=[-1.25,1.25] \times[-1.25,1.25]$. We compute the errors of the solution in $L_{2}(0, T ; \Omega)$, denoted by $E_{2}, L_{\infty}\left(0, T ; L_{2}(\Omega)\right)$ denoted by $E_{\infty}$ and for the gradient of the solution in $L_{2}(0, T ; \Omega)$ denoted by $E G_{2}$ and $L_{\infty}\left(0, T ; L_{2}(\Omega)\right)$ denoted by $E G_{\infty}$. We refined the grid from $n=10$ to $n=320$, taking for $b$ a large value, e.g. greater than $C 320$ where $C$ is a range of initial condition.

Example 1. In this example we compare a numerical solution with the exact solution

$$
u(x, y, t)=\frac{x^{2}+y^{2}}{2}+t
$$

to the equation

$$
\frac{u_{t}}{\sqrt{|\nabla u|^{2}+0.5}}-\operatorname{div}\left(\frac{\nabla u}{\sqrt{|\nabla u|^{2}+0.5}}\right)=-\frac{0.5}{\left(x^{2}+y^{2}+0.5\right)^{\frac{3}{2}}},
$$

with non-homogeneous (exact) Dirichlet boundary conditions in time interval $[0, T]=[0,0.3125]$.

The results for the semi-implicit and fully implicit schemes are summarised in tables below. In this example time step $\tau$ fulfils the relation $\tau=h^{2}$ (natural for solving parabolic PDEs), where $h=\frac{1}{n}$ is the size of the side of finite volume. For computing the linear system in every discrete time step we use the Gauss-Seidel iterative solver. The iterations are stopped when the square of relative residual drops below a prescribed tolerance TOL. For the semi-implicit scheme and $T O L=10^{-15}$ we need about 30-40 iterations in each time step and the results are presented in Table 1. For the fully-implicit scheme and $T O L=10^{-12}$, for the Gauss-Seidel solver as well as for nonlinear iterative procedure, we need about 20-30 Gauss-Seidel iterations and about 30-40 nonlinear iterations. The results are presented in Table 2. We test also $\alpha$ scheme (39)-(40)-(41)-(42) with $\alpha=0.6, T O L=10^{-10}$ both for semi-implicit and fully implicit schemes, the results are given in Tables 3 and 4 . We can observe that the fully implicit scheme is about three times more precise than semi-implicit one. The $\alpha$ scheme slightly improves the accuracy of the semi-implicit one and does not significantly modify that of the fully implicit scheme. All schemes have the same experimental order of convergence which is, for the coupling $\tau=h^{2}$, equal to 2 for the solution error and equal to 1 for the gradient error. We also tested the $\alpha$ schemes with $\alpha=0.5$ instead of 0.6 and $\tau=h$. We got a very poor order of convergence close to 0.8 for the semi-implicit scheme and a divergent behaviour for the fully implicit one, that is compatible with the expectations of the end of Section 3 .

\begin{tabular}{|c|c|c|c|c|c|c|c|c|c|}
\hline$n$ & $\tau$ & $E_{2}$ & $E O C$ & $E_{\infty}$ & $E O C$ & $E G_{2}$ & $E O C$ & $E G_{\infty}$ & $E O C$ \\
\hline 10 & $6.25 \mathrm{e}-02$ & $5.006 \mathrm{e}-02$ & - & $1.753 \mathrm{e}-01$ & - & $4.513 \mathrm{e}-01$ & - & $9.622 \mathrm{e}-01$ & - \\
\hline 20 & $1.5625 \mathrm{e}-02$ & $1.806 \mathrm{e}-02$ & 1.471 & $4.312 \mathrm{e}-02$ & 2.024 & $2.232 \mathrm{e}-01$ & 1.016 & $4.412 \mathrm{e}-01$ & 1.125 \\
\hline 40 & $3.90625 \mathrm{e}-03$ & $5.405 \mathrm{e}-03$ & 1.740 & $1.244 \mathrm{e}-02$ & 1.793 & $9.712 \mathrm{e}-02$ & 1.199 & $1.831 \mathrm{e}-01$ & 1.269 \\
\hline 80 & $9.76563 \mathrm{e}-04$ & $1.456 \mathrm{e}-03$ & 1.892 & $3.267 \mathrm{e}-03$ & 1.929 & $4.079 \mathrm{e}-02$ & 1.253 & $7.431 \mathrm{e}-02$ & 1.3001 \\
\hline 160 & $2.44141 \mathrm{e}-04$ & $3.739 \mathrm{e}-04$ & 1.962 & $8.285 \mathrm{e}-04$ & 1.979 & $1.757 \mathrm{e}-02$ & 1.215 & $3.162 \mathrm{e}-02$ & 1.233 \\
\hline 320 & $6.10352 \mathrm{e}-05$ & $9.451 \mathrm{e}-05$ & 1.984 & $2.083 \mathrm{e}-04$ & 1.992 & $7.932 \mathrm{e}-03$ & 1.147 & $1.422 \mathrm{e}-02$ & 1.153 \\
\hline
\end{tabular}

Table 1: Example 1, error reports and EOCs for the semi-implicit scheme 


\begin{tabular}{|c|c|c|c|c|c|c|c|c|c|}
\hline$n$ & $\tau$ & $E_{2}$ & $E O C$ & $E_{\infty}$ & $E O C$ & $E G_{2}$ & $E O C$ & $E G_{\infty}$ & EOC \\
\hline 10 & $6.25 \mathrm{e}-02$ & $3.885 \mathrm{e}-02$ & - & $7.253 \mathrm{e}-02$ & - & $3.401 \mathrm{e}-01$ & - & $6.615 \mathrm{e}-01$ & - \\
\hline 20 & $1.5625 \mathrm{e}-02$ & $1.010 \mathrm{e}-02$ & 1.944 & $1.834 \mathrm{e}-02$ & 1.983 & $1.511 \mathrm{e}-01$ & 1.171 & $2.772 \mathrm{e}-01$ & 1.255 \\
\hline 40 & $3.90625 \mathrm{e}-03$ & $2.532 \mathrm{e}-03$ & 1.995 & $4.551 \mathrm{e}-03$ & 2.011 & $6.686 \mathrm{e}-02$ & 1.176 & $1.202 \mathrm{e}-01$ & 1.205 \\
\hline 80 & $9.76563 \mathrm{e}-04$ & $6.315 \mathrm{e}-04$ & 2.003 & $1.132 \mathrm{e}-03$ & 2.007 & $3.077 \mathrm{e}-02$ & 1.120 & $5.509 \mathrm{e}-02$ & 1.126 \\
\hline 160 & $2.44141 \mathrm{e}-04$ & $1.574 \mathrm{e}-04$ & 2.004 & $2.821 \mathrm{e}-04$ & 2.004 & $1.465 \mathrm{e}-02$ & 1.071 & $2.622 \mathrm{e}-02$ & 1.071 \\
\hline 320 & $6.10352 \mathrm{e}-05$ & $3.931 \mathrm{e}-05$ & 2.002 & $7.043 \mathrm{e}-05$ & 2.002 & $7.135 \mathrm{e}-03$ & 1.038 & $1.277 \mathrm{e}-02$ & 1.038 \\
\hline
\end{tabular}

Table 2: Example 1, error reports and EOCs for the fully implicit scheme

\begin{tabular}{|c|c|c|c|c|c|c|c|c|c|}
\hline$n$ & $\tau$ & $E_{2}$ & $E O C$ & $E_{\infty}$ & $E O C$ & $E G_{2}$ & $E O C$ & $E G_{\infty}$ & $E O C$ \\
\hline 10 & $6.25 \mathrm{e}-02$ & $2.570 \mathrm{e}-02$ & - & $5.113 \mathrm{e}-02$ & - & $3.241 \mathrm{e}-01$ & - & $6.298 \mathrm{e}-01$ & - \\
\hline 20 & $1.5625 \mathrm{e}-02$ & $8.367 \mathrm{e}-03$ & 1.620 & $1.879 \mathrm{e}-02$ & 1.445 & $1.386 \mathrm{e}-01$ & 1.225 & $2.680 \mathrm{e}-01$ & 1.232 \\
\hline 40 & $3.90625 \mathrm{e}-03$ & $2.512 \mathrm{e}-03$ & 1.737 & $5.562 \mathrm{e}-03$ & 1.756 & $5.739 \mathrm{e}-02$ & 1.272 & $1.063 \mathrm{e}-01$ & 1.339 \\
\hline 80 & $9.76563 \mathrm{e}-04$ & $6.832 \mathrm{e}-04$ & 1.879 & $1.482 \mathrm{e}-03$ & 1.908 & $2.363 \mathrm{e}-02$ & 1.280 & $4.282 \mathrm{e}-02$ & 1.312 \\
\hline 160 & $2.44141 \mathrm{e}-04$ & $1.770 \mathrm{e}-04$ & 1.948 & $3.801 \mathrm{e}-04$ & 1.963 & $1.009 \mathrm{e}-02$ & 1.230 & $1.812 \mathrm{e}-02$ & 1.241 \\
\hline 320 & $6.10352 \mathrm{e}-05$ & $4.497 \mathrm{e}-05$ & 1.978 & $9.608 \mathrm{e}-05$ & 1.984 & $4.522 \mathrm{e}-03$ & 1.158 & $8.099 \mathrm{e}-03$ & 1.162 \\
\hline
\end{tabular}

Table 3: Example 1, error reports and EOCs for the $\alpha$ semi-implicit scheme, $\alpha=0.6$

Example 2. Now we use the exact solution $[27]$

$$
u(x, y, t)=\min \left\{\frac{x^{2}+y^{2}-1}{2}+t, 0\right\}
$$

to the level set equation (4)

$$
\frac{u_{t}}{|\nabla u|}-\operatorname{div}\left(\frac{\nabla u}{|\nabla u|}\right)=0,
$$

with zero Dirichlet boundary conditions, in time interval $[0, T]=[0,0.3125]$. The initial condition and exact and numerically computed solution $(n=40)$ are plotted in Figures $1,2$.

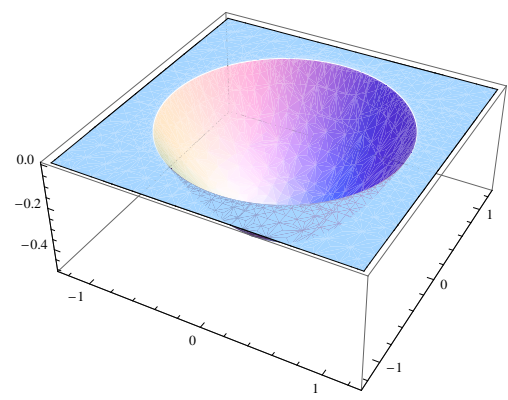

Figure 1: Example 2: Initial condition

In this example we have to use the Evans-Spruck type regularisation, the solution contains flat regions and a singular circular curve with gradient jump, so we cannot expect second order accuracy. However as we see from the tables, the numerical schemes converge also in this singular case and naturally, EOC is equal (or close to) 1 for the solution error. Again the fully implicit scheme is about three times more precise than semi-implicit one in the solution error, but not in the gradient error. First we have chosen constant value of the regularisation parameter $a=10^{-6}$ and $\tau=h^{2}$, the results for semi-implicit, fully implicit and $\alpha$ semi-implicit schemes are given in Table 5, 6 and 7. Then we used also the coupling $a=h^{2}$, the results for semi-implicit and fully implicit schemes are in Tables 8, 9, this coupling provides a slightly better EOC. 


\begin{tabular}{|c|c|c|c|c|c|c|c|c|c|}
\hline$n$ & $\tau$ & $E_{2}$ & $E O C$ & $E_{\infty}$ & $E O C$ & $E G_{2}$ & $E O C$ & $E G_{\infty}$ & $E O C$ \\
\hline 10 & $6.25 \mathrm{e}-02$ & $2.962 \mathrm{e}-02$ & - & $5.820-02$ & - & $2.189 \mathrm{e}-01$ & - & $4.703 \mathrm{e}-01$ & - \\
\hline 20 & $1.5625 \mathrm{e}-02$ & $8.325 \mathrm{e}-03$ & 1.831 & $1.715 \mathrm{e}-02$ & 1.763 & $8.358 \mathrm{e}-02$ & 1.390 & $1.852 \mathrm{e}-01$ & 1.344 \\
\hline 40 & $3.90625 \mathrm{e}-03$ & $2.255 \mathrm{e}-03$ & 1.884 & $4.661 \mathrm{e}-03$ & 1.880 & $3.580 \mathrm{e}-02$ & 1.223 & $7.788 \mathrm{e}-02$ & 1.250 \\
\hline 80 & $9.76563 \mathrm{e}-04$ & $5.878 \mathrm{e}-04$ & 1.940 & $1.210 \mathrm{e}-03$ & 1.946 & $1.662 \mathrm{e}-02$ & 1.107 & $3.477 \mathrm{e}-02$ & 1.164 \\
\hline 160 & $2.44141 \mathrm{e}-04$ & $1.499 \mathrm{e}-04$ & 1.971 & $3.076 \mathrm{e}-04$ & 1.975 & $8.043 \mathrm{e}-03$ & 1.047 & $1.625 \mathrm{e}-02$ & 1.097 \\
\hline 320 & $6.10352 \mathrm{e}-05$ & $3.785 \mathrm{e}-05$ & 1.986 & $7.753 \mathrm{e}-05$ & 1.988 & $3.963 \mathrm{e}-03$ & 1.021 & $7.821 \mathrm{e}-03$ & 1.055 \\
\hline
\end{tabular}

Table 4: Example 1, error reports and EOCs for the $\alpha$ fully implicit scheme, $\alpha=0.6$
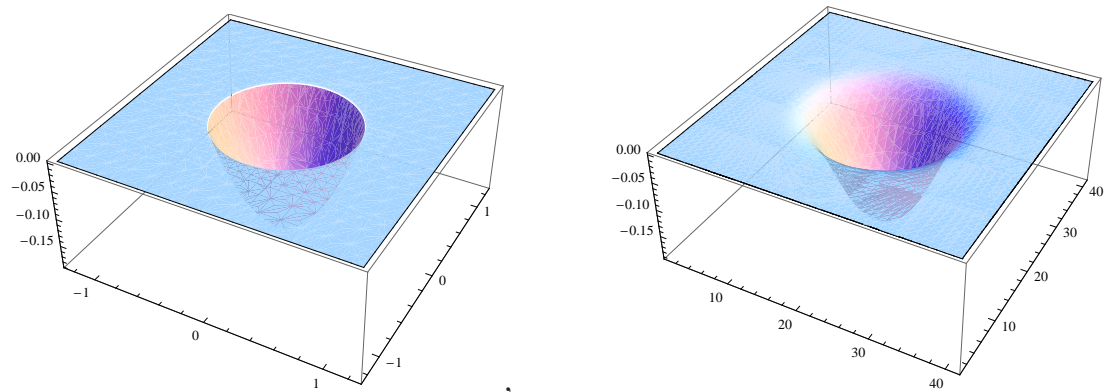

Figure 2: Example 2, the exact (left) and numerical solutions (right) at time 0.3125 .

\section{Conclusions}

The properties proven in this paper show that the fully implicit and the semi-implicit schemes have interesting mathematical and numerical properties. But it now remains to show that these convergence properties can also be extended to the non-regularised situation where $f$ and $g$ are given by (4).

Acknowledgements. The authors thank Professor Guy Barles for interesting and fruitful discussions.

\section{Appendix}

Lemma 6.1 Let $b$ be a continuous strictly increasing function from $\mathbb{R}$ to $\mathbb{R}$. Let $\left(\beta_{n}\right)_{n \in \mathbb{N}}$ be a sequence in $\mathbb{R}$ and $\beta \in R$ such that $\left(b\left(\beta_{n}\right)-b(\beta)\right)\left(\beta_{n}-\beta\right) \longrightarrow 0$ as $n \longrightarrow \infty$. Then, $\beta_{n} \longrightarrow \beta$ as $n \longrightarrow \infty$.

Proof. We first remark that the mapping $s \mapsto(b(\gamma+s \delta)-b(\gamma)) \delta$ is increasing, for all $\delta, \gamma \in \mathbb{R}$. This will be used to prove that the sequence $\left(\beta_{n}\right)_{n \in \mathbb{N}}$ is bounded. Indeed, if the sequence $\left(\beta_{n}\right)_{n \in \mathbb{N}}$ is unbounded, we can assume, up to a subsequence, that $\left|\beta_{n}\right| \longrightarrow \infty$ as $n \longrightarrow \infty$ and then, once again up to a subsequence, that $\left|\beta_{n}-\beta\right| \geq 1$ for all $n \in \mathbb{N}$ and $\frac{\beta_{n}-\beta}{\left|\beta_{n}-\beta\right|} \longrightarrow \gamma$ as $n \longrightarrow \infty$ (for some $\gamma \in \mathbb{R}$ with $|\gamma|=1$ ). Therefore, one has:

$$
\left(b\left(\beta_{n}\right)-b(\beta)\right)\left(\beta_{n}-\beta\right) \geq\left(b\left(\beta+\frac{\beta_{n}-\beta}{\left|\beta_{n}-\beta\right|}\right)-b(\beta)\right) \frac{\beta_{n}-\beta}{\left|\beta_{n}-\beta\right|} .
$$

Then, passing to the limit as $n \longrightarrow \infty$,

$$
0=\lim _{n \longrightarrow \infty}\left(b\left(\beta_{n}\right)-b(\beta)\right)\left(\beta_{n}-\beta\right) \geq(b(\beta+\gamma)-b(\beta)) \cdot \gamma>0 .
$$

which is impossible.

Since the sequence $\left(\beta_{n}\right)_{n \in \mathbb{N}}$ is bounded, we can assume, up to a subsequence, that $\beta_{n} \longrightarrow \gamma$, as $n \longrightarrow \infty$, for some $\gamma \in \mathbb{R}$. Then, since $\left(b\left(\beta_{n}\right)-b(\beta)\right)\left(\beta_{n}-\beta\right) \longrightarrow 0$, one has $(b(\gamma)-b(\beta))(\gamma-\beta)=0$, which gives $\gamma=\beta$ and the convergence of the whole sequence $\left(\beta_{n}\right)_{n \in \mathbb{N}}$ to $\beta$ follows. 


\begin{tabular}{|c|c|c|c|c|c|c|c|c|c|}
\hline$n$ & $\tau$ & $E_{2}$ & $E O C$ & $E_{\infty}$ & $E O C$ & $E G_{2}$ & $E O C$ & $E G_{\infty}$ & EOC \\
\hline 10 & $6.25 \mathrm{e}-02$ & $5.109 \mathrm{e}-02$ & - & $1.156 \mathrm{e}-01$ & - & $2.653 \mathrm{e}-01$ & - & $5.393 \mathrm{e}-01$ & - \\
\hline 20 & $1.5625 \mathrm{e}-02$ & $2.965 \mathrm{e}-02$ & 0.785 & $7.054 \mathrm{e}-02$ & 0.713 & $2.174 \mathrm{e}-01$ & 0.287 & $4.522 \mathrm{e}-01$ & 0.254 \\
\hline 40 & $3.90625 \mathrm{e}-03$ & $1.596 \mathrm{e}-02$ & 0.892 & $3.836 \mathrm{e}-02$ & 0.879 & $1.719 \mathrm{e}-01$ & 0.339 & $3.442 \mathrm{e}-01$ & 0.393 \\
\hline 80 & $9.76563 \mathrm{e}-04$ & $8.393 \mathrm{e}-03$ & 0.930 & $2.002 \mathrm{e}-02$ & 0.938 & $1.381 \mathrm{e}-01$ & 0.317 & $2.709 \mathrm{e}-01$ & 0.346 \\
\hline 160 & $2.44141 \mathrm{e}-04$ & $4.341 \mathrm{e}-03$ & 0.951 & $1.037 \mathrm{e}-02$ & 0.950 & $1.096 \mathrm{e}-01$ & 0.332 & $2.139 \mathrm{e}-01$ & 0.341 \\
\hline 320 & $6.10352 \mathrm{e}-05$ & $2.270 \mathrm{e}-03$ & 0.935 & $5.433 \mathrm{e}-03$ & 0.932 & $8.7131 \mathrm{e}-02$ & 0.332 & $1.693 \mathrm{e}-01$ & 0.338 \\
\hline
\end{tabular}

Table 5: Example 2, error reports and EOCs for semi-implicit scheme, $a=10^{-6}$

\begin{tabular}{|c|c|c|c|c|c|c|c|c|c|}
\hline$n$ & $\tau$ & $E_{2}$ & $E O C$ & $E_{\infty}$ & $E O C$ & $E G_{2}$ & $E O C$ & $E G_{\infty}$ & EOC \\
\hline 10 & $6.25 \mathrm{e}-02$ & $1.583 \mathrm{e}-02$ & - & $3.304 \mathrm{e}-02$ & - & $1.683 \mathrm{e}-01$ & - & $3.512 \mathrm{e}-01$ & - \\
\hline 20 & $1.5625 \mathrm{e}-02$ & $8.462 \mathrm{e}-03$ & 0.904 & $1.938 \mathrm{e}-02$ & 0.770 & $1.559 \mathrm{e}-01$ & 0.111 & $3.658 \mathrm{e}-01$ & -0.059 \\
\hline 40 & $3.90625 \mathrm{e}-03$ & $4.442 \mathrm{e}-03$ & 0.930 & $9.959 \mathrm{e}-03$ & 0.961 & $1.367 \mathrm{e}-01$ & 0.186 & $3.004 \mathrm{e}-01$ & 0.284 \\
\hline 80 & $9.76563 \mathrm{e}-04$ & $2.404 \mathrm{e}-03$ & 0.886 & $5.333 \mathrm{e}-03$ & 0.901 & $1.126 \mathrm{e}-01$ & 0.280 & $2.542 \mathrm{e}-01$ & 0.241 \\
\hline 160 & $2.44141 \mathrm{e}-04$ & $1.320 \mathrm{e}-03$ & 0.865 & $3.033 \mathrm{e}-03$ & 0.814 & $9.231 \mathrm{e}-02$ & 0.287 & $1.865 \mathrm{e}-01$ & 0.447 \\
\hline 320 & $6.10352 \mathrm{e}-05$ & $8.110 \mathrm{e}-04$ & 0.702 & $1.920 \mathrm{e}-03$ & 0.660 & $7.603 \mathrm{e}-02$ & 0.280 & $1.490 \mathrm{e}-01$ & 0.324 \\
\hline
\end{tabular}

Table 6: Example 2, error reports and EOCs for fully implicit scheme, $a=10^{-6}$

Lemma 6.2 Let $\left(F_{n}\right)_{n \in \mathbb{N}}$ be a sequence nonnegative functions in $L^{1}(\Omega)$. Let $F \in L^{1}(\Omega)$ be such that $F_{n} \longrightarrow F$ a.e. in $\Omega$ and $\int_{\Omega} F_{n}(x) \mathrm{d} x \longrightarrow \int_{\Omega} F(x) \mathrm{d} x$, as $n \longrightarrow \infty$. Then, $F_{n} \longrightarrow F$ in $L^{1}(\Omega)$ as $n \longrightarrow \infty$.

Proof. The proof of this lemma is very classical. Applying the Dominated Convergence Theorem to the sequence $\left(F-F_{n}\right)^{+}$leads to $\int_{\Omega}\left(F(x)-F_{n}(x)\right)^{+} \mathrm{d} x \longrightarrow 0$ as $n \longrightarrow \infty$. Then, since $\left|F-F_{n}\right|=$ $2\left(F-F_{n}\right)^{+}-\left(F-F_{n}\right)$, we conclude that $F_{n} \longrightarrow F$ in $L^{1}(\Omega)$ as $n \longrightarrow \infty$.

Theorem 6.1 (A variant of Ascoli's theorem) Let $\Omega$ be a polyhedral open bounded connected subset of $\mathbb{R}^{d}$, with $d \in \mathbb{N} \backslash\{0\}$ and $T>0$. Let $u_{0} \in H_{0}^{1}(\Omega)$ be given. Let $\left(u_{m}, \mathcal{D}_{m}, \tau_{m}\right)_{m \in \mathbb{N}}$ be a sequence such that, for all $m \in \mathbb{N},\left(\mathcal{D}_{m}, \tau_{m}\right)$ is a space-time discretisation of $\Omega \times(0, T)$ in the sense of Definition 2.2, $u_{m} \in H_{\mathcal{D}_{m}, \tau_{m}}$ and $h_{\mathcal{D}_{m}}$ and $\tau_{m}$ tend to 0 as $m \longrightarrow \infty$. For all $m \in \mathbb{N}$, setting $\mathcal{D}=\mathcal{D}_{m}$ and $\tau=\tau_{m}$, we define the functions $u_{\mathcal{D}, \tau}(x, t)$, for all $t \in[0, T]$ and a.e. $x \in \Omega$ by

$$
\begin{aligned}
& u_{\mathcal{D}, \tau}(x, 0)=u_{p}^{0}=\frac{1}{|p|} \int_{p} u_{0}(x) \mathrm{d} x, \\
& \left.\left.u_{\mathcal{D}, \tau}(x, t)=u_{p}^{n+1}, \text { for a.e. } x \in p, \forall t \in\right] n \tau,(n+1) \tau\right], \forall p \in \mathcal{M}, \forall n=0, \ldots, N_{T},
\end{aligned}
$$

and the function $z_{\mathcal{D}, \tau}$ by

$$
\left.z_{\mathcal{D}, \tau}(x, t)=\frac{u_{p}^{n+1}-u_{p}^{n}}{\tau}, \text { for a.e. } x \in p, \quad \text { for a.e. } t \in\right] n \tau,(n+1) \tau[, \forall p \in \mathcal{M}, \forall n \in \mathbb{N},
$$

with $u_{p}^{0}$ defined by (64). We assume that there exists $C>0$ (hence independent of $m$ ) such that $\left\|u_{m}^{n+1}\right\|_{1, \mathcal{D}_{m}} \leq C$ for all $n=0, \ldots, N_{T m}$ and $\left\|z_{\mathcal{D}_{m}, \tau_{m}}\right\|_{L^{2}(\Omega \times(0, T))}^{2} \leq C$.

Then there exists $\bar{u} \in C^{0}\left(0, T ; L^{2}(\Omega)\right)$ with $\bar{u}(\cdot, 0)=u_{0}$ and a subsequence of $\left(u_{m}, \mathcal{D}_{m}, \tau_{m}\right)_{m \in \mathbb{N}}$, again denoted $\left(u_{m}, \mathcal{D}_{m}, \tau_{m}\right)_{m \in \mathbb{N}}$, such that

$$
\lim _{m \rightarrow \infty} \sup _{t \in[0, T]}\left\|u_{\mathcal{D}_{m}, \tau_{m}}(t)-u(t)\right\|_{L^{2}(\Omega)}=0 .
$$

Proof. 


\begin{tabular}{|c|c|c|c|c|c|c|c|c|c|}
\hline$n$ & $\tau$ & $E_{2}$ & $E O C$ & $E_{\infty}$ & $E O C$ & $E G_{2}$ & $E O C$ & $E G_{\infty}$ & EOC \\
\hline 10 & $6.25 \mathrm{e}-02$ & $5.074 \mathrm{e}-02$ & - & $1.091 \mathrm{e}-01$ & - & $2.722 \mathrm{e}-01$ & - & $5.404 \mathrm{e}-01$ & - \\
\hline 20 & $1.5625 \mathrm{e}-2$ & $2.985 \mathrm{e}-02$ & 0.765 & $6.891 \mathrm{e}-02$ & 0.663 & $2.227 \mathrm{e}-01$ & 0.259 & $4.587 \mathrm{e}-01$ & 0.236 \\
\hline 40 & $3.90625 \mathrm{e}-03$ & $1.582 \mathrm{e}-02$ & 0.916 & $3.748 \mathrm{e}-02$ & 0.879 & $1.743 \mathrm{e}-01$ & 0.384 & $3.471 \mathrm{e}-01$ & 0.402 \\
\hline 80 & $9.76563 \mathrm{e}-04$ & $8.345 \mathrm{e}-03$ & 0.923 & $1.973 \mathrm{e}-02$ & 0.925 & $1.386 \mathrm{e}-01$ & 0.331 & $2.712 \mathrm{e}-01$ & 0.356 \\
\hline 160 & $2.44141 \mathrm{e}-04$ & $4.323 \mathrm{e}-03$ & 0.949 & $1.027 \mathrm{e}-02$ & 0.942 & $1.098 \mathrm{e}-01$ & 0.336 & $2.137 \mathrm{e}-01$ & 0.343 \\
\hline 320 & $6.10352 \mathrm{e}-05$ & $2.264 \mathrm{e}-03$ & 0.933 & $5.403 \mathrm{e}-03$ & 0.927 & $8.717 \mathrm{e}-02$ & 0.333 & $1.692 \mathrm{e}-01$ & 0.337 \\
\hline
\end{tabular}

Table 7: Example 2, error reports and EOCs for $\alpha$ semi-implicit scheme with $\alpha=0.6, a=10^{-6}$

\begin{tabular}{|c|c|c|c|c|c|c|c|c|c|}
\hline$n$ & $\tau$ & $E_{2}$ & $E O C$ & $E_{\infty}$ & $E O C$ & $E G_{2}$ & $E O C$ & $E G_{\infty}$ & EOC \\
\hline 10 & $6.25 \mathrm{e}-02$ & $5.717 \mathrm{e}-02$ & - & $1.288 \mathrm{e}-01$ & - & $2.602 \mathrm{e}-01$ & - & $5.252 \mathrm{e}-01$ & - \\
\hline 20 & $1.5625 \mathrm{e}-02$ & $3.159 \mathrm{e}-02$ & 0.856 & $7.513 \mathrm{e}-02$ & 0.780 & $2.143 \mathrm{e}-01$ & 0.280 & $4.436 \mathrm{e}-01$ & 0.244 \\
\hline 40 & $3.90625 \mathrm{e}-03$ & $1.634 \mathrm{e}-02$ & 0.951 & $3.929 \mathrm{e}-02$ & 0.935 & $1.711 \mathrm{e}-01$ & 0.325 & $3.422 \mathrm{e}-01$ & 0.374 \\
\hline 80 & $9.76563 \mathrm{e}-04$ & $8.385 \mathrm{e}-03$ & 0.963 & $2.001 \mathrm{e}-02$ & 0.973 & $1.381 \mathrm{e}-01$ & 0.310 & $2.710 \mathrm{e}-01$ & 0.337 \\
\hline 160 & $2.44141 \mathrm{e}-04$ & $4.253 \mathrm{e}-03$ & 0.979 & $1.013 \mathrm{e}-02$ & 0.985 & $1.101 \mathrm{e}-01$ & 0.445 & $2.146 \mathrm{e}-01$ & 0.336 \\
\hline 320 & $6.10352 \mathrm{e}-05$ & $2.150 \mathrm{e}-03$ & 0.984 & $5.101 \mathrm{e}-03$ & 0.987 & $8.789 \mathrm{e}-02$ & 0.206 & $1.707 \mathrm{e}-01$ & 0.666 \\
\hline
\end{tabular}

Table 8: Example 2, error reports and EOCs for semi-implicit scheme, $a=h^{2}$

We first remark that, for $m \in \mathbb{N}$, denoting $\mathcal{D}=\mathcal{D}_{m}$ and $\tau=\tau_{m}$ and for all $\left.\left.t_{1} \in\right]\left(n_{1}-1\right) \tau, n_{1} \tau\right]$ and $\left.\left.t_{2} \in\right]\left(n_{2}-1\right) \tau, n_{2} \tau\right]$, for $n_{1}, n_{2}=0, \ldots, N_{T}$ with $n_{2}>n_{1}$ and a.e. $x \in p$, we have

$$
\left(u_{\mathcal{D}, \tau}\left(x, t_{1}\right)-u_{\mathcal{D}, \tau}\left(x, t_{2}\right)\right)^{2} \leq\left(\sum_{n=n_{1}}^{n_{2}-1}\left|u_{p}^{n+1}-u_{p}^{n}\right|\right)^{2} \leq\left(n_{2}-n_{1}\right) \tau \sum_{n=n_{1}}^{n_{2}-1} \frac{\left(u_{p}^{n+1}-u_{p}^{n}\right)^{2}}{\tau} .
$$

Hence we get that

$$
\int_{\Omega}\left(u_{\mathcal{D}_{m}, \tau_{m}}\left(x, t_{1}\right)-u_{\mathcal{D}_{m}, \tau_{m}}\left(x, t_{2}\right)\right)^{2} \mathrm{~d} x \leq C\left(\left|t_{2}-t_{1}\right|+\tau_{m}\right), \forall t_{1}, t_{2} \in[0, T], \forall m \in \mathbb{N} .
$$

The proof can now follow that of Ascoli's theorem. Let $\left(t_{k}\right)_{k \in \mathbb{N}}$ be a dense sequence in $[0, T]$. For $t=t_{0}$, if $t_{O}>0$, we can use the result given in [17] since $\left\|u_{m}^{n+1}\right\|_{1, \mathcal{D}_{m}} \leq C$ for all $n=0, \ldots, N_{T}$ :

$$
\left\|u_{\mathcal{D}_{m}, \tau_{m}}\left(\cdot+\xi, t_{0}\right)-u_{\mathcal{D}_{m}, \tau_{m}}\left(\cdot, t_{0}\right)\right\|_{L^{2}(\Omega \times(0, T))}^{2} \leq C|\xi|\left(|\xi|+4 h_{\mathcal{D}_{m}}\right), \forall \xi \in \mathbb{R}^{d} .
$$

Hence we can extract, thanks to Kolmogorov's theorem, a subsequence of $\left(u_{m}, \mathcal{D}_{m}, \tau_{m}\right)_{m \in \mathbb{N}}$, denoted by $\left(u_{\psi_{0}(m)}, \mathcal{D}_{\psi_{0}(m)}, \tau_{\psi_{0}(m)}\right)_{m \in \mathbb{N}}$, where $\psi_{0}$ is an increasing injection from $\mathbb{N}$ to $\mathbb{N}$, such that $u_{\mathcal{D}_{\psi_{0}(m)}, \tau_{\psi_{0}(m)}}\left(\cdot, t_{0}\right)$ converges in $L^{2}(\Omega)$ to some function. Note also that, thanks to $(64), u_{\mathcal{D}_{m}, \tau_{m}}(\cdot, 0)$ converges in $L^{2}(\Omega)$ to $u_{0}$. Similarly, for $t=t_{1}$, one extracts, again thanks to Kolmogorov's theorem, a subsequence of $\left(u_{\psi_{0}(m)}, \mathcal{D}_{\psi_{0}(m)}, \tau_{\psi_{0}(m)}\right)_{m \in \mathbb{N}}$, denoted $\left(u_{\psi_{1}(m)}, \mathcal{D}_{\psi_{1}(m)}, \tau_{\psi_{1}(m)}\right)_{m \in \mathbb{N}}$ such that $u_{\mathcal{D}_{\psi_{1}(m)}, \tau_{\psi_{1}(m)}}\left(\cdot, t_{1}\right)$ converges in $L^{2}(\Omega)$ to some function. We reproduce this mechanism by induction for all $k \in \mathbb{N}$, allowing to consider the diagonal sequence $\left(u_{\psi_{m}(m)}, \mathcal{D}_{\psi_{m}(m)}\right)_{m \in \mathbb{N}}$, which is then such that $u_{\mathcal{D}_{\psi_{m}(m)}, \tau_{\psi_{m}(m)}}\left(\cdot, t_{k}\right)$ converges in $L^{2}(\Omega)$ as $m \rightarrow \infty$ for all $k \in \mathbb{N}$ (recall that the sequence $\left(u_{\psi_{m}(m)}, \mathcal{D}_{\psi_{m}(m)}, \tau_{\psi_{m}(m)}\right)_{m \in \mathbb{N}}$ is extracted from $\left.\left(u_{\psi_{k}(m)}, \mathcal{D}_{\psi_{k}(m)}, \tau_{\psi_{k}(m)}\right)_{m \in \mathbb{N}, m \geq k}\right)$. We now denote, for simplicity, $\left(u_{m}, \mathcal{D}_{m}, \tau_{m}\right)_{m \in \mathbb{N}}$ instead of $\left(u_{\psi_{m}(m)}, \mathcal{D}_{\psi_{m}(m)}, \tau_{\psi_{m}(m)}\right)_{m \in \mathbb{N}}$.

Then the property $(67)$ allows to show that, for all $t \in \mathbb{R}_{+},\left(u_{\mathcal{D}_{m}, \tau_{m}}(t)\right)_{m \in \mathbb{N}}$ is a Cauchy sequence in $L^{2}(\Omega)$. Indeed, for $\left.\varepsilon \in\right] 0,1\left[\right.$, one first chooses $k \in \mathbb{N}$ such that $\left|t-t_{k}\right| \leq \varepsilon^{2}$, then $n_{0} \in \mathbb{N}$ such that $\tau_{m} \leq \varepsilon^{2}$ for all $n \geq n_{0}$, and $\left\|u_{\mathcal{D}_{n}, \tau_{n}}\left(t_{k}\right)-u_{\mathcal{D}_{p}, \tau_{p}}\left(t_{k}\right)\right\|_{L^{2}(\Omega)} \leq \varepsilon$ for all $n, p \geq n_{0}$. The inequality $\left\|u_{\mathcal{D}_{n}, \tau_{n}}(t)-u_{\mathcal{D}_{p}, \tau_{p}}(t)\right\|_{L^{2}(\Omega)} \leq\left\|u_{\mathcal{D}_{n}, \tau_{n}}(t)-u_{\mathcal{D}_{n}, \tau_{n}}\left(t_{k}\right)\right\|_{L^{2}(\Omega)}+\left\|u_{\mathcal{D}_{n}, \tau_{n}}\left(t_{k}\right)-u_{\mathcal{D}_{p}, \tau_{p}}\left(t_{k}\right)\right\|_{L^{2}(\Omega)}+\| u_{\mathcal{D}_{p}, \tau_{p}}\left(t_{k}\right)-$ $u_{\mathcal{D}_{p}, \tau_{p}}(t) \|_{L^{2}(\Omega)} \leq(2 \sqrt{2 C}+1) \varepsilon$ for all $n, p \geq n_{0}$ follows. 


\begin{tabular}{|c|c|c|c|c|c|c|c|c|c|}
\hline$n$ & $\tau$ & $E_{2}$ & $E O C$ & $E_{\infty}$ & $E O C$ & $E G_{2}$ & $E O C$ & $E G_{\infty}$ & $\mathrm{EOC}$ \\
\hline 10 & $6.25 \mathrm{e}-02$ & $3.455 \mathrm{e}-02$ & - & $8.102 \mathrm{e}-02$ & - & $1.967 \mathrm{e}-01$ & - & $3.917 \mathrm{e}-01$ & - \\
\hline 20 & $1.5625 \mathrm{e}-2$ & $1.199 \mathrm{e}-02$ & 1.527 & $2.936 \mathrm{e}-02$ & 1.465 & $1.631 \mathrm{e}-01$ & 0.270 & $3.888 \mathrm{e}-01$ & 0.010 \\
\hline 40 & $3.90625 \mathrm{e}-03$ & $5.032 \mathrm{e}-03$ & 1.253 & $1.165 \mathrm{e}-02$ & 1.333 & $1.394 \mathrm{e}-01$ & 0.227 & $3.071 \mathrm{e}-01$ & 0.341 \\
\hline 80 & $9.76563 \mathrm{e}-04$ & $2.397 \mathrm{e}-03$ & 1.070 & $5.321 \mathrm{e}-03$ & 1.131 & $1.123 \mathrm{e}-01$ & 0.311 & $2.372 \mathrm{e}-01$ & 0.372 \\
\hline 160 & $2.44141 \mathrm{e}-04$ & $1.185 \mathrm{e}-03$ & 1.017 & $2.636 \mathrm{e}-03$ & 1.013 & $9.079 \mathrm{e}-02$ & 0.307 & $1.822 \mathrm{e}-01$ & 0.381 \\
\hline 320 & $6.10352 \mathrm{e}-05$ & $5.937 \mathrm{e}-04$ & 0.997 & $1.330 \mathrm{e}-03$ & 0.987 & $7.284 \mathrm{e}-02$ & 0.318 & $1.432 \mathrm{e}-01$ & 0.348 \\
\hline
\end{tabular}

Table 9: Example 2, error reports and EOCs for fully implicit scheme, $a=h^{2}$

One then defines, for all $t \in \mathbb{R}_{+}, \bar{u}(t)$ as the limit of $\left(u_{\mathcal{D}_{m}, \tau_{m}}(t)\right)_{m \in \mathbb{N}}$. Passing to the limit $m \rightarrow \infty$ in (67) provides

$$
\left\|\bar{u}\left(t_{2}\right)-\bar{u}\left(t_{1}\right)\right\|_{L^{2}(\Omega)}^{2} \leq C\left|t_{2}-t_{1}\right|, \forall t_{1}, t_{2} \in[0, T], \forall T>0,
$$

which shows that $u \in C^{0}\left(\mathbb{R}_{+} ; L^{2}(\Omega)\right)$. Then (66) is again an easy consequence of (67). Indeed, let $T \geq 0$ and $\varepsilon>0$ be given. Since, for all $k=0, \ldots,\left\lfloor T / \varepsilon^{2}\right\rfloor$ (where $\lfloor x\rfloor$ denotes the greater integer lower of equal to $x$ ), the sequence $\left(u_{\mathcal{D}_{m}, \tau_{m}}\left(k \varepsilon^{2}\right)\right)_{m \in \mathbb{N}}$ converges to $u\left(k \varepsilon^{2}\right)$, let $n_{0} \in \mathbb{N}$ be such that $\left\|u_{\mathcal{D}_{m}, \tau_{m}}\left(k \varepsilon^{2}\right)-u\left(k \varepsilon^{2}\right)\right\|_{L^{2}(\Omega)} \leq \varepsilon$ for all $k=0, \ldots,\left\lfloor T / \varepsilon^{2}\right\rfloor$ and all $m \geq n_{0}$, and such that $\tau_{m} \leq \varepsilon^{2}$ for all $m \geq n_{0}$. Then, for all $t \in[0, T]$ and $m \geq n_{0}$, letting $k=\left\lfloor t / \varepsilon^{2}\right\rfloor$, we get using (68) and (67), $\left\|\bar{u}(t)-u_{\mathcal{D}_{m}, \tau_{m}}(t)\right\|_{L^{2}(\Omega)} \leq\left\|\bar{u}(t)-\bar{u}\left(k \varepsilon^{2}\right)\right\|_{L^{2}(\Omega)}+\left\|\bar{u}\left(k \varepsilon^{2}\right)-u_{\mathcal{D}_{m}, \tau_{m}}\left(k \varepsilon^{2}\right)\right\|_{L^{2}(\Omega)}+\| u_{\mathcal{D}_{m}, \tau_{m}}\left(k \varepsilon^{2}\right)-$ $u_{\mathcal{D}_{m}, \tau_{m}}(t) \|_{L^{2}(\Omega)} \leq(\sqrt{C}+1+\sqrt{2 C}) \varepsilon$, which concludes the proof of $(66)$.

\section{References}

[1] L. Alvarez, F. Guichard, P. L. Lions, and J. M. Morel. Axioms and fundamental equations of image processing. Archive for Rat. Mech. Anal., 123:200-257, 1993.

[2] B. Andreianov, F. Boyer, and F. Hubert. Discrete duality finite volume schemes for Leray-Lions-type elliptic problems on general 2D meshes. Numer. Methods Partial Differential Equations, 23(1):145$195,2007$.

[3] S. B. Angenent and M. E. Gurtin. Multiphase thermomechanics with an interfacial structure 2. Evolution of an isothermal interface. Arch. Rat. Mech. Anal., 108:323-391, 1989.

[4] G. Barles and P.E. Souganidis. Convergence of approximation schemes for fully nonlinear second order equations. Asymptotic Analysis, 4(3):271-283, 1991.

[5] V. Caselles, R. Kimmel, and G. Sapiro. Geodesic active contours. International Journal of Computer Vision, 22:61-79, 1997.

[6] F. Catté, P. L. Lions, J. M. Morel, and T. Coll. Image selective smoothing and edge detection by nonlinear diffusion. SIAM J. Num. Anal., 29:182-193, 1992.

[7] Y. Chen, Y. Giga, and S. Goto. Uniqueness and existence of viscosity solutions of generalized mean curvature flow equation. J. Differential Geometry, 33:749-786, 1991.

[8] Y. Chen, B.C. Vemuri, and L. Wang. Image denoising and segmentation via nonlinear diffusion. Computers and Mathematics with Applications, 39:131-149, 2000.

[9] S. Corsaro, K. Mikula, A. Sarti, and F. Sgallari. Semi-implicit co-volume method in 3D image segmentation. SIAM Journal on Scientific Computing, 28(6):2248-2265, 2006.

[10] K. Deckelnick and G. Dziuk. Error estimates for a semi-implicit fully discrete finite element scheme for the mean curvature flow of graphs. Interfaces and Free Boundaries, 2:341-359, 2000. 
[11] K. Deckelnick and G. Dziuk. Convergence of numerical schemes for the approximation of level set solutions to mean curvature flow. In Numerical methods for viscosity solutions and applications (Heraklion, 1999), volume 59 of Ser. Adv. Math. Appl. Sci., pages 77-93. World Sci. Publ., River Edge, NJ, 2001.

[12] K. Deckelnick and G. Dziuk. Numerical aproximation of mean curvature fow of gaphs and level sets. In L. Ambrosio, K. Deckelnick, G. Dziuk, M. Mimura, V. A. Solonnikov, and H. M. Soner, editors, Mathematical aspects of evolving interfaces, pages 53-87. Springer, Berlin-Heidelberg-New York, 2003.

[13] O. Drblíková, M. Komorníkova, M. Remešíková, P. Bourgine, K. Mikula, N. Peyriéras, and A. Sarti. Estimate of the cell number growth rate using PDE methods of image processing and time series analysis. Journal of Electrical Engineering, 58(7/s):86-92, 2007.

[14] J. Droniou. Finite volume schemes for fully non-linear elliptic equations in divergence form. $M 2 A N$ Math. Model. Numer. Anal., 40(6):1069-1100, 2006.

[15] L. C. Evans and J. Spruck. Motion of level sets by mean curvature I. J. Differential Geometry, 33:635-681, 1991.

[16] R. Eymard and T. Gallouët. H-convergence and numerical schemes for elliptic equations. SIAM Journal on Numerical Analysis, 41(2):539-562, 2003.

[17] R. Eymard, T. Gallouët, and R. Herbin. Finite volume methods. In P. G. Ciarlet and J.-L. Lions, editors, Techniques of Scientific Computing, Part III, Handbook of Numerical Analysis, VII, pages 713-1020. North-Holland, Amsterdam, 2000.

[18] R. Eymard, T. Gallouët, and R. Herbin. Finite volume approximation of elliptic problems and convergence of an approximate gradient. Appl. Numer. Math., 37(1-2):31-53, 2001.

[19] R. Eymard, T. Gallouët, and R. Herbin. Cell centred discretisation of non linear elliptic problems on general multidimensional polyhedral grids. to be published in J. Numer. Math., 2009.

[20] R. Eymard, T. Gallouët, and R. Herbin. Discretisation of heterogeneous and anisotropic diffusion problems on general nonconforming meshes. SUSHI: a scheme using stabilization and hybrid interfaces. to be published in IMAJNA, 2009.

[21] A. Handlovičová and K. Mikula. Stability and consistency of the semi-implicit co-volume scheme for the regularized mean curvature flow equation in level set formulation. Applications of Mathematics, $2: 105-129,2008$.

[22] A. Handlovičová, K. Mikula, and F. Sgallari. Semi-implicit complementary volume scheme for solving level set like equations in image processing and curve evolution. Numer. Math., 93:675-695, 2003.

[23] S. Kichenassamy, A. Kumar, P. Olver, A. Tannenbaum, and A. Yezzi. Conformal curvature flows: from phase transitions to active vision. Arch. Rational Mech. Anal., 134:275-301, 1996.

[24] K. Mikula, N. Peyriéras, M. Remešíková, and A. Sarti. 3D embryogenesis image segmentation by the generalized subjective surface method using the finite volume technique. In R. Eymard and J.-M. Hérard, editors, Finite Volumes for Complex Applications V, pages 585-592. Wiley, 2008.

[25] K. Mikula and N. Ramarosy. Semi-implicit finite volume scheme for solving nonlinear diffusion equations in image processing. Numer. Math., 89(3):561-590, 2001.

[26] K. Mikula, A. Sarti, and F. Sgallari. Co-volume method for riemannian mean curvature flow in subjective surfaces multiscale segmentation. Comput. Visual. Sci., 9(1):23-31, 2006. 
[27] A.M. Oberman. A convergent monotone difference scheme for motion of level sets by mean curvature. Numer. Math., 99(2):365-379, 2004.

[28] S. Osher and J. Sethian. Fronts propagating with curvature dependent speed: algorithm based on hamilton-jacobi formulation. J. Comput. Phys., 79:12-49, 1988.

[29] A. Sarti, R. Malladi, and J.A. Sethian. Subjective surfaces: A method for completing missing boundaries. Proceedings of the National Academy of Sciences of the United States of America, 12(97):6258-6263, 2000.

[30] A. Schmidt. Computation of three dimensional dendrites with finite elements. Journal of Computational Physics, 125(2):293-312, 1996.

[31] N. J. Walkington. Algorithms for computing motion by mean curvature. SIAM J. Numer. Anal., 33(6):2215-2238, 1996. 